\title{
Snakebite Envenoming a Challenging Diagnosis for the Forensic Pathologist: A Systematic Review
}

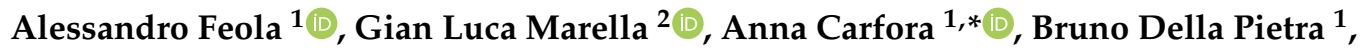 \\ Pierluca Zangani ${ }^{1}$ and Carlo Pietro Campobasso ${ }^{1}$ (D) \\ 1 Department Experimental Medicine, University of Campania “Luigi Vanvitelli”, via Luciano Armanni 5, \\ 80138 Naples, Italy; alessandro.feola@unicampania.it (A.F.); bruno.dellapietra@unicampania.it (B.D.P.); \\ pierluca.zangani@unicampania.it (P.Z.); carlopietro.campobasso@unicampania.it (C.P.C.) \\ 2 Department of Surgical Sciences, University of Rome "Tor Vergata”, via Montpellier 1, 00133 Rome, Italy; \\ glmarella@gmail.com \\ * Correspondence: anna.carfora@unicampania.it; Tel.: +39-081-566-6017
}

Received: 1 October 2020; Accepted: 30 October 2020; Published: 3 November 2020

\begin{abstract}
Snakebite envenoming (SBE) is a public health issue in developing countries. The estimated annual global incidence of snakebites is about 5.4 million snakebites per year, resulting from 1.8 to 2.7 million cases of SBE and from 81,000 to 138,000 deaths with 400,000 survivors suffering permanent physical and psychological disabilities. There are more than 3000 species of snakes around the world: 600 are venomous and over 200 are considered to be medically important because of their clinical effects. The severity of SBE depends on several factors among which bite localization, snake's size, condition of glands and teeth, bite angle and bite duration, the microflora of the snake's mouth and victim's skin, age of the victim, weight, health status, and victim's activity after a bite. Snake venoms are mixtures of protein families, and each of these families contains many different toxins or toxin isoforms. Based on their effects, snake venoms can be classified as hemotoxic, neurotoxic, or cytotoxic and they can all act together involving multiple tissues and organs. When the bite is fatal, the mechanism of death is primarily related to the paralysis of respiratory muscles, which causes asphyxia and hypoxic-ischemic encephalopathy, but also anaphylactic shock, hemorrhagic shock, cardiomyopathy, acute tubular necrosis (ATN). The purpose of this literature review is to evaluate epidemiological and post-mortem examination findings in fatal SBEs in order to better understand the pathophysiological mechanisms, thus helping pathologists in defining the correct diagnosis.
\end{abstract}

Keywords: snakebite envenomation; autopsy; toxicology; neglected tropical disease

Key Contribution: (1) SBE is a potentially life-threatening disease resulting from the injection of a mixture of different toxins (venom) that particularly afflicts the poorest people living in developing countries with the worst quality of life. (2) The diagnosis of SBE related death is challenging for the forensic pathologist since autopsy findings are mostly non-specific. (3) Pathologic changes commonly found are consistent with a rapid death such as multi-visceral congestion, edema, and petechial hemorrhages. (4) The key elements for SBE diagnosis are still represented by investigation of the bite mark and circumstantial data.

\section{Introduction}

SBE is a potentially life-threatening disease resulting from the injection of a mixture of different toxins (venom) that particularly afflicts the poorest people living in developing countries with the worst quality of life [1]. In 2017, the World Health Organization (WHO) listed SBE as a priority neglected tropical disease (NTD). WHO reports 5.4 million snakebites per year, resulting from 1.8 to 
2.7 million cases of SBE and from 81,000 to 138,000 deaths with 400,000 survivors suffering permanent physical and psychological disabilities [1,2]. The promptness of the therapy is a key element for a good outcome after SBE. However, given the socio-cultural and economic context of the territories, effective treatment is often delayed due to the lack of hospital infrastructure, the non-availability of a species-specific antivenom or delay in its injection caused by the not prompt identification of the offending snake, and the use of traditional healers or popular remedies [3,4]. Pathogenesis induced by snake venoms is complex and characterized by local and systemic alterations represented mostly by bleeding, dermo/myonecrosis, inflammation, and coagulation disorders. Mechanisms of toxicity remain poorly understood because the venoms are complex mixtures of components that induce different effects [5]. Snake venom can be detected post-mortem through many toxicological ways, such as bioassays, radioimmunoassays (RIA), fluorescence immunoassays, immunoelectrophoresis, ELISA (enzyme-linked immunosorbent assay) [6]. Cytotoxins, neurotoxins, and hemotoxins are commonly identified depending on the species of the offending snake.

There are more than 3000 species of snakes around the world: 600 are venomous and over 200 are considered to be medically important because of their clinical effects [7]. New species of snakes are still being discovered, and many species formerly recognized as being widespread have been found to comprise multiple separate species [8]. Although the majority of snake species do not have venom and kill their prey by squeezing them, snakes are classified by WHO guidelines $[8,9]$ based on production, control, and regulation of snake antivenom immunoglobulins and according to risk assessment: in category 1 (highest medical importance) there are highly venomous snakes that are common or widespread and cause numerous snakebites, resulting in high levels of morbidity, disability or mortality; in category 2 (secondary medical importance) there are highly venomous snakes capable of causing morbidity, disability or death, for which exact epidemiological or clinical data may be lacking; and/or are less frequently implicated. The aim of this study is to evaluate retrospectively the characteristics of fatal SBE reported in the literature.

\section{Results}

\subsection{Characteristics of Eligible Studies}

The search of PubMed, Scopus, Web of Science, and Google Scholar databases provided a total of 263 articles. After adjusting for duplicates and reviewing titles and abstracts, 228 studies were discarded. Finally, only 35 papers reporting, in total, 56 cases of fatal SBE satisfied the inclusion criteria. A flowchart depicting the selection of studies according to PRISMA standards [10] is reported in Figure 1. Details of the 35 papers satisfying the inclusion criteria are summarized in Table 1. 
Table 1. Summary of the items recorded from the 35 papers included in the retrospective study.

\begin{tabular}{|c|c|c|c|c|c|c|c|c|c|c|}
\hline References & Country & $\begin{array}{c}\text { Victim } \\
\text { Age/Sex }\end{array}$ & $\begin{array}{l}\text { Work- } \\
\text { Related }\end{array}$ & $\begin{array}{c}\text { Hospital } \\
\text { Admission } \\
J \\
\text { Antivenom } \\
\end{array}$ & $\begin{array}{l}\text { Time Interval } \\
\text { Bite-Death }\end{array}$ & $\begin{array}{l}\text { Snake } \\
\text { Family }\end{array}$ & $\begin{array}{c}\text { Bite Area } \\
\text { Site } \\
\text { Macroscopical Findings }\end{array}$ & $\begin{array}{c}\text { Bite Area } \\
\text { Histological Findings }\end{array}$ & $\begin{array}{l}\text { Autopsy } \\
\text { Macroscopical Findings }\end{array}$ & $\begin{array}{c}\text { Autopsy } \\
\text { Histological Pattern }\end{array}$ \\
\hline $\begin{array}{l}\text { Barraviera Bet al., } \\
\quad 1989[11]\end{array}$ & Brazil & $60 / \mathrm{M}$ & n.a. & Yes/Yes & $4-5 \mathrm{~d}$ & $\begin{array}{c}\text { Rattlesnake } \\
\text { Viperidae }\end{array}$ & $\begin{array}{l}\text { Right leg. } \\
\text { Ulceration covered with } \\
\text { a blood-stained scab. }\end{array}$ & $\begin{array}{l}\text { Local edema and } \\
\text { inflammatory signs. } \\
\text { Focal myonecrosis. }\end{array}$ & n.a. & K: acute tubular necrosis \\
\hline $\begin{array}{c}\text { Barraviera B et } \\
\text { al.,1989 [11] }\end{array}$ & Brazil & $59 / \mathrm{M}$ & n.a. & Yes/Yes & $5 \mathrm{~d}$ & $\begin{array}{c}\text { Rattlesnake } \\
\text { Viperidae }\end{array}$ & $\begin{array}{c}\text { Right leg. } \\
\text { Two punctiform } \\
\text { erythematous lesions. }\end{array}$ & $\begin{array}{l}\text { No signs of } \\
\text { inflammation. }\end{array}$ & n.a. & Li: parenchymal necrosis. \\
\hline $\begin{array}{l}\text { Benvenuti LA et } \\
\text { al., } 2003[12]\end{array}$ & Brazil & $36 / \mathrm{F}$ & n.a. & Yes; No & $45 \mathrm{~min}$ & $\begin{array}{c}\text { Bothrops jararacussu } \\
\text { Viperidae }\end{array}$ & $\begin{array}{l}\text { Left ankle. } \\
\text { Two puncture wounds } \\
25 \mathrm{~mm} \text { apart. } \\
\text { Ecchymosis on the left } \\
\text { leg along the great } \\
\text { saphenous vein. }\end{array}$ & $\begin{array}{l}\text { Local hemorrhage, } \\
\text { thrombosis, and } \\
\text { coagulative necrosis of } \\
\text { the subcutaneous and } \\
\text { muscular tissue. }\end{array}$ & L: hemorrhage. & $\begin{array}{l}\text { PG: normal. } \\
\text { H: fibrosis and interstitial } \\
\text { hemorrhage. } \\
\text { L: extensive alveolar hemorrhage } \\
\text { and edema with numerous platelet } \\
\text { thrombi in capillaries and } \\
\text { venules. DIC. }\end{array}$ \\
\hline $\begin{array}{l}\text { Blaylock RSM et } \\
\text { al., 2002 [13] }\end{array}$ & South Africa & $40 / \mathrm{M}$ & Yes & Yes; Yes & $1 \mathrm{~h} 15 \min$ & $\begin{array}{l}\text { Puff Adder } \\
\text { (Bitis Arientas) } \\
\text { Viperidae }\end{array}$ & $\begin{array}{l}\text { Left wrist. } \\
\text { Single puncture wound. }\end{array}$ & $\begin{array}{l}\text { Extensive hemorrhage } \\
\text { between muscle bundles } \\
\text { and connective tissue. }\end{array}$ & $\begin{array}{l}\text { H: myocardium soft and } \\
\text { flabby with hemorrhagic } \\
\text { RA. } \\
\text { L: edema and congestion. } \\
\text { Li; K: pale aspect. }\end{array}$ & n.a. \\
\hline $\begin{array}{l}\text { Blumenthal R et } \\
\text { al., } 2019[14]\end{array}$ & South Africa & $27 / \mathrm{M}$ & Yes & Yes; Yes & $3 \mathrm{~d}$ & $\begin{array}{c}\text { Black Mamba } \\
\text { (Dendroaspis polylepis) } \\
\text { Elapidae }\end{array}$ & $\begin{array}{l}\text { Right hand. } \\
\text { Single puncture mark. }\end{array}$ & $\begin{array}{l}\text { Subcutaneous } \\
\text { hemorrhage. } \\
\text { Minimal skin necrosis } \\
\text { and hemorrhage at the } \\
\text { puncture site. }\end{array}$ & $\begin{array}{l}\text { Diffuse fresh } \\
\text { hemorrhages around the } \\
\text { vocal cords, the } \\
\text { epiglottis, and in the LV. } \\
\text { H: petechiae overlying } \\
\text { the anterior and } \\
\text { posterior epicardial. }\end{array}$ & $\begin{array}{l}\text { B: hypoxic-ischemic } \\
\text { encephalopathy. } \\
\text { H: diffuse hemorrhages. } \\
\text { L: fibinosuppurative } \\
\text { consolidation. }\end{array}$ \\
\hline $\begin{array}{c}\text { Chen Let al., } 2013 \\
\text { [15] }\end{array}$ & China & $43 / \mathrm{M}$ & n.a. & Yes (at the end); n.a. & $5 \mathrm{~d}$ & $\begin{array}{l}\text { Agkistrodom } \\
\text { halysViperidae }\end{array}$ & $\begin{array}{c}\text { Right foot. } \\
0.3 \mathrm{~cm}-\text { long laceration } \\
\text { made of two pairs of } \\
\text { thin stripes. Right foot } \\
\text { swollen and ecchymotic. }\end{array}$ & $\begin{array}{l}\text { Subcutaneous } \\
\text { hemorrhage and } \\
\text { cellulitis. }\end{array}$ & $\begin{array}{l}\text { Diffuse edema and } \\
\text { congestion. }\end{array}$ & $\begin{array}{l}\text { K: Toxic nephropathy, proximal } \\
\text { tubular epithelial degeneration, } \\
\text { and necrosis, protein and pigment } \\
\text { cast in the distal tubules. }\end{array}$ \\
\hline $\begin{array}{l}\text { Chen L et al., } 2013 \\
\text { [15] }\end{array}$ & China & $4 / \mathrm{M}$ & n.a. & Yes; n.a. & $2 \mathrm{~d}$ & $\begin{array}{l}\text { Agkistrodom } \\
\text { halysViperidae }\end{array}$ & $\begin{array}{c}\text { Right foot. } \\
1.2 \times 0.5 \mathrm{~cm} \\
\text { snakebite wound. }\end{array}$ & n.a. & $\begin{array}{l}\text { Diffuse edema and } \\
\text { congestion.S: } \\
\text { anemic aspect. }\end{array}$ & $\begin{array}{c}\text { B, T, K: congestion. } \\
\text { H, L: edema and congestion. }\end{array}$ \\
\hline $\begin{array}{l}\text { Curry SC et al., } \\
1985[16]\end{array}$ & USA & $44 / \mathrm{F}$ & No & Yes; Yes & $15 \mathrm{~d}$ & $\begin{array}{l}\text { Rattlesnake } \\
\text { Viperidae }\end{array}$ & $\begin{array}{l}\text { Right calf. } \\
\text { Two fang marks } \\
\text { surrounded ba an area } \\
\text { of discoloration. }\end{array}$ & $\begin{array}{l}\text { Area of tissue disruption } \\
\text { leading toward a vin. } \\
\text { Small eschar over the } \\
\text { site of fang penentration } \\
\text { through epidermidis. }\end{array}$ & $\begin{array}{l}\text { B, Li, S: enlarged and } \\
\text { congested. } \\
\text { H: lightly dilated and } \\
\text { flabby; superficial } \\
\text { hemorrhages. } \\
\text { L: expanded and } \\
\text { indurated. }\end{array}$ & $\begin{array}{l}\text { B: focal hemorrhages in the } \\
\text { leptomeninges. } \\
\text { L: severe ARDS. } \\
\text { K: eosinophilic proteinaceous casts } \\
\text { in small tubules. }\end{array}$ \\
\hline $\begin{array}{l}\text { Karlawad M et al., } \\
\quad 2017[17]\end{array}$ & India & $75 / \mathrm{M}$ & Yes & Yes; Yes & $3 \mathrm{~h}$ & n.a. & $\begin{array}{l}\text { Scrotum. } \\
\text { Two punctured wounds } \\
\text { surrounded by an area } \\
\text { of discoloration. }\end{array}$ & $\begin{array}{c}\text { Ulcerated stratified } \\
\text { squamous epithelium } \\
\text { with sub-eppithelial tissue } \\
\text { showing congested } \\
\text { blood vessess } \\
\text { and edema. }\end{array}$ & Diffuse congestion. & $\begin{array}{l}\text { L: interstitial and intra-alveolar } \\
\text { edema with rupture of alveoli. } \\
\text { H: unremarkable. }\end{array}$ \\
\hline
\end{tabular}


Table 1. Cont.

\begin{tabular}{|c|c|c|c|c|c|c|c|c|c|c|}
\hline References & Country & $\begin{array}{c}\text { Victim } \\
\text { Age/Sex }\end{array}$ & $\begin{array}{c}\text { Work- } \\
\text { Related }\end{array}$ & $\begin{array}{c}\text { Hospital } \\
\text { Admission } \\
J \\
\text { Antivenom } \\
\end{array}$ & $\begin{array}{l}\text { Time Interval } \\
\text { Bite-Death }\end{array}$ & $\begin{array}{l}\text { Snake } \\
\text { Family }\end{array}$ & $\begin{array}{c}\text { Bite Area } \\
\text { Site } \\
\text { Macroscopical Findings }\end{array}$ & $\begin{array}{c}\text { Bite Area } \\
\text { Histological Findings }\end{array}$ & $\begin{array}{l}\text { Autopsy } \\
\text { Macroscopical Findings }\end{array}$ & $\begin{array}{c}\text { Autopsy } \\
\text { Histological Pattern }\end{array}$ \\
\hline $\begin{array}{c}\text { Keith J et al., } 2012 \\
{[18]}\end{array}$ & Australia & 69/F & No & Yes; Yes & $2 \mathrm{~d}$ & $\begin{array}{c}\text { Australian Brown Snake } \\
\text { (Pseudonaja textilis) } \\
\text { Elapidade }\end{array}$ & Foot. & n.a. & $\begin{array}{l}\text { B: swollen with tonsillar } \\
\text { herniation and V-shaped } \\
\text { hemorrhage within } \\
\text { the cerebellum. }\end{array}$ & Unremarkable. \\
\hline $\begin{array}{l}\text { Kitchens CS et al., } \\
\quad 1987[19]\end{array}$ & USA & $67 / \mathrm{M}$ & No & Yes; Yes & $2 \mathrm{~d} 12 \mathrm{~h}$ & $\begin{array}{l}\text { Canebrake Rattlesnake } \\
\text { (Crotalus Horridus } \\
\text { Atricaudatus) } \\
\text { Viperidae }\end{array}$ & $\begin{array}{l}\text { Left Hand. } \\
\text { Two puncture wounds. }\end{array}$ & $\begin{array}{l}\text { Rhabdomyolysis. The } \\
\text { left thenar muscle } \\
\text { disclosed a sparse } \\
\text { infiltration of PMNs. }\end{array}$ & $\begin{array}{l}\text { M: soft consistency } \\
\text { underlying the bite. }\end{array}$ & $\begin{array}{l}\text { H: patchy myocardial necrosis. } \\
\text { L: hemorrhagic pulmonary edema; } \\
\text { small scattered pulmonary thrombi } \\
\text { consistent with terminal DIC. } \\
\text { K: massive acute tubular necrosis. } \\
\text { M: rhabdomyolysis of the } \\
\text { skeletal muscles. }\end{array}$ \\
\hline $\begin{array}{l}\text { Malbranque S et } \\
\text { al., 2008 [20] }\end{array}$ & Martinique & $74 / \mathrm{M}$ & Yes & Yes; Yes & $10 \mathrm{~d}$ & $\begin{array}{l}\text { Fer-de-Lance (Bothrops } \\
\text { lanceolatus) Viperidae }\end{array}$ & $\begin{array}{l}\text { Left elbow. } \\
\text { Fang puncture marks } 20 \\
\text { mm apart surrounded } \\
\text { by a swollen area. }\end{array}$ & n.a. & $\begin{array}{l}\text { Serous effusions in the } \\
\text { peritoneal, pericardial, } \\
\text { and pleural cavities. } \\
\text { B: edema with petechiae. } \\
\text { H: infarcts of different } \\
\text { ages and sizes. } \\
\text { L: edematous and } \\
\text { congested. }\end{array}$ & $\begin{array}{l}\text { B: ischemic changes; thrombotic } \\
\text { lesions were confined to the } \\
\text { sub-arachnoid space. } \\
\text { H: Fibrinous pericarditis and } \\
\text { scattered ischemic foci in the LV. } \\
\text { L: thrombotic lesion within the } \\
\text { small pulmonary arteries and the } \\
\text { capillaries of the } \\
\text { inter-alveolar septa. } \\
\text { K: thrombotic lesion within the } \\
\text { small renal inter-lobular vessels } \\
\text { and, to a localized extent. } \\
\text { I: thrombotic lesion in the small } \\
\text { intestine and colon. }\end{array}$ \\
\hline $\begin{array}{l}\text { Malhotra P et al., } \\
2005[21]\end{array}$ & India & $27 / \mathrm{F}$ & n.a. & Yes; Yes & n.a. & n.a. & $\begin{array}{l}\text { Left foot. } \\
\text { Fang marks. }\end{array}$ & n.a. & Unremarkable. & $\begin{array}{l}\text { B: extensive perivascular } \\
\text { demyelination and } \\
\text { limphocyte cuffing. } \\
\text { K: acuthe tubular necrosis. }\end{array}$ \\
\hline $\begin{array}{l}\text { Marsh N, et al., } \\
\quad 2007[22]\end{array}$ & $\begin{array}{c}\text { USA } \\
\text { (captivity) }\end{array}$ & $33 / \mathrm{F}$ & No & No; No & n.a. & $\begin{array}{l}\text { Gaboon viper (Bitis } \\
\text { gabonica) Viperidae }\end{array}$ & $\begin{array}{l}\text { Left hand. } \\
\text { Two puncture wounds } \\
\text { 11-12 mm apart } \\
\text { surrounded by a swollen } \\
\text { and bloody area. }\end{array}$ & n.a. & $\begin{array}{l}\text { Ecchymoses of the head, } \\
\text { trunk and extremities, } \\
\text { the periorbital areas, } \\
\text { and left orolaryngeal/ } \\
\text { mandibular angle. } \\
\text { L: hemorrhagic } \\
\text { appearance and } \\
\text { congestion. } \\
\end{array}$ & n.a. \\
\hline $\begin{array}{l}\text { McGarity BH et } \\
\text { al., } 1991[23]\end{array}$ & Australia & $40 / \mathrm{M}$ & n.a. & Yes; Yes & $2 \mathrm{~d}$ & $\begin{array}{l}\text { Tiger snake (Notechis } \\
\text { scutatus) Elapidae }\end{array}$ & Left heel. & n.a. & $\begin{array}{l}\text { B: edematous; right } \\
\text { tentorial and subfackial } \\
\text { herniation; massive right } \\
\text { intracerebral } \\
\text { hemorrhage, with } \\
\text { rupture into the right } \\
\text { basal ganglia and } \\
\text { cerebellum. }\end{array}$ & n.a. \\
\hline
\end{tabular}


Table 1. Cont.

\begin{tabular}{|c|c|c|c|c|c|c|c|c|c|c|}
\hline References & Country & $\begin{array}{c}\text { Victim } \\
\text { Age/Sex }\end{array}$ & $\begin{array}{l}\text { Work- } \\
\text { Related }\end{array}$ & $\begin{array}{c}\text { Hospital } \\
\text { Admission } \\
\quad J \\
\text { Antivenom } \\
\end{array}$ & $\begin{array}{c}\text { Time Interval } \\
\text { Bite-Death }\end{array}$ & $\begin{array}{l}\text { Snake } \\
\text { Family }\end{array}$ & $\begin{array}{c}\text { Bite Area } \\
\text { Site } \\
\text { Macroscopical Findings }\end{array}$ & $\begin{array}{c}\text { Bite Area } \\
\text { Histological Findings }\end{array}$ & $\begin{array}{l}\text { Autopsy } \\
\text { Macroscopical Findings }\end{array}$ & $\begin{array}{c}\text { Autopsy } \\
\text { Histological Pattern }\end{array}$ \\
\hline $\begin{array}{l}\text { Milani R Jr et al., } \\
\quad 1997[24]\end{array}$ & Brazil & $3 / \mathrm{F}$ & No & Yes; Yes & $18 \mathrm{~h} 40 \mathrm{~min}$ & $\begin{array}{l}\text { Jararacuçu (Bothrops } \\
\text { jararacussu) Viperidae }\end{array}$ & $\begin{array}{l}\text { Left thigh. } \\
\text { Three widely spaced } \\
\text { fang puncture marks. }\end{array}$ & $\begin{array}{c}\text { Extensive } \\
\text { rhabdomyolysis with } \\
\text { hemorrhagic foci. } \\
\text { Hemorrhage and } \\
\text { necrosis in the dermis } \\
\text { and hemorrhage in } \\
\text { subcutaneous fat near } \\
\text { the site. }\end{array}$ & B: edema. & $\begin{array}{l}\text { L: hemorrhages with inflammatory } \\
\text { infiltration of neutrophils in septa } \\
\text { and alveoli and deposition of } \\
\text { intra-alveolar fibrin. } \\
\text { Li: hepatic sinusoids and portal } \\
\text { tracts infiltrated with PMNs. } \\
\text { P: fatty necrosis with hemorrhage } \\
\text { of the parenchymal cells. }\end{array}$ \\
\hline $\begin{array}{l}\text { Milani R Jr et al., } \\
\quad 1997[24]\end{array}$ & Brazil & $65 / \mathrm{M}$ & n.a. & Yes; Yes & $4 \mathrm{~d} 9 \mathrm{~h}$ & $\begin{array}{l}\text { Jararacuçu (Bothrops } \\
\text { jararacussu) Viperidae }\end{array}$ & $\begin{array}{l}\text { Left calf. } \\
\text { Two fang punctures } \\
\text { surrounded by a ring of } \\
\text { bruising. }\end{array}$ & $\begin{array}{l}\text { Necrosis of muscle fibers } \\
\text { and interstitial } \\
\text { hemorrlage in the } \\
\text { region of the wound. }\end{array}$ & $\begin{array}{l}\text { B: subarachnoid } \\
\text { petechiae and edema. }\end{array}$ & $\begin{array}{l}\text { B: ischemic necrosis of the mucosa } \\
\text { and in small cortical and meningeal } \\
\text { vessels with local meningeal } \\
\text { hemorrhage and cerebral edema. } \\
\text { Li: fatty (alcoholic) liver with } \\
\text { evidence of shock. } \\
\text { I: fibrin thrombi in small } \\
\text { sub-mucosal vessels. } \\
\text { AG: focal hemorrhages in the } \\
\text { medulla, with depletion of the } \\
\text { zona fasciculata. } \\
\text { K: acute tubular necrosis DIC. }\end{array}$ \\
\hline $\begin{array}{l}\text { Moar JJ et al., } 2016 \\
\text { [25] }\end{array}$ & South Africa & $23 / \mathrm{M}$ & Yes & Yes; No & Several hours & $\begin{array}{l}\text { Rinkhal (Hemachatus } \\
\text { haemachatus) Elapidae }\end{array}$ & $\begin{array}{l}\text { Right forearm. } \\
\text { Two bite marks each } 3 \\
\mathrm{~mm} \text { in diameter and } 1.5 \\
\mathrm{~cm} \text { apart. }\end{array}$ & $\begin{array}{l}\text { Displaced and disrupted } \\
\text { fragments of adnexal } \\
\text { structures into the } \\
\text { depths of the wound, } \\
\text { together with } \\
\text { hemorrhaging into the } \\
\text { surrounding } \\
\text { interstitium, and } \\
\text { compression of the } \\
\text { collagen bundles of the } \\
\text { dermis with collagen } \\
\text { displacement and } \\
\text { disruption. }\end{array}$ & $\begin{array}{l}\text { B, L: edema and } \\
\text { petechial hemorrhages. }\end{array}$ & $\begin{array}{l}\text { B: Extravasation of erythrocytes } \\
\text { into the surrounding } \\
\text { Virchow-Robin spaces. } \\
\text { H: in LV marked interstitial } \\
\text { vascular dilatation and congestion } \\
\text { with focal subendocardial papillary } \\
\text { muscle fibrosis. } \\
\text { L ARDS. } \\
\text { Li: portal vessels congestion and } \\
\text { widening by reticulin and a portal } \\
\text { triadititis. } \\
\text { S: sinusidalal dilation and } \\
\text { congestion; mantle zone } \\
\text { yyperplasia. } \\
\text { K: interstitial vascular and } \\
\text { glomerular capillary loop } \\
\text { dilatation and congestion with a } \\
\text { chronic inflammatory cell infiltrate. }\end{array}$ \\
\hline $\begin{array}{c}\text { Namal } \\
\text { Rathnaayaka } \\
\text { RMMK et al., 2018 } \\
\text { [26] } \\
\end{array}$ & Sri Lanka & $42 / \mathrm{M}$ & Yes & Yes; No & $16 \mathrm{~d}$ & $\begin{array}{l}\text { Hump nose Viper } \\
\text { (Hypnale Hypnale) } \\
\text { Viperidae }\end{array}$ & Right foot. & n.a. & $\begin{array}{l}\text { B: a small infarct (1.5 } \\
\mathrm{cm}-1 \mathrm{~cm} \text { ) in the basal } \\
\text { ganglia. } \\
\text { H: reduced lumen of the } \\
\text { left coronary artery. }\end{array}$ & $\begin{array}{l}\text { L: hemorrhages. } \\
\text { K: acute tubular necrosis. } \\
\text { H: normal. }\end{array}$ \\
\hline $\begin{array}{c}\text { Namal } \\
\text { Rathnayaka } \\
\text { RMMK etal., } 2019 \\
\text { [27] }\end{array}$ & Sri Lanka & $66 / \mathrm{M}$ & No & Yes; No & $4 \mathrm{~d}$ & $\begin{array}{c}\text { Hump nose Viper } \\
\text { (Hypnale Hypnale) } \\
\text { Viperidae }\end{array}$ & $\begin{array}{l}\text { Left foot. } \\
\text { Two fang punctures. }\end{array}$ & n.a. & B, H, L: hemorrhages. & L: edema and hemorrhage. \\
\hline $\begin{array}{c}\text { Namal } \\
\text { Rathnayaka } \\
\text { RMMK etal., } 2017 \\
\text { [28] }\end{array}$ & Sri Lanka & $43 / \mathrm{M}$ & No & Yes; Yes & $11 \mathrm{~d}$ & $\begin{array}{l}\text { Russell's viper (Daboia } \\
\text { russelii) Viperidae }\end{array}$ & $\begin{array}{l}\text { Right foot. } \\
\text { Swelling of the leg. }\end{array}$ & n.a. & $\begin{array}{l}\text { B: infarcted area of } \\
\text { parietal lobes. } \\
\text { K: petechial } \\
\text { hemorrhages. }\end{array}$ & $\begin{array}{l}\text { B: hemorrhages, thrombi, and } \\
\text { congested blood vessels. } \\
\text { H, L, K: normal. }\end{array}$ \\
\hline
\end{tabular}


Table 1. Cont

\begin{tabular}{|c|c|c|c|c|c|c|c|c|c|c|}
\hline References & Country & $\begin{array}{c}\text { Victim } \\
\text { Age/Sex }\end{array}$ & $\begin{array}{c}\text { Work- } \\
\text { Related }\end{array}$ & $\begin{array}{c}\text { Hospital } \\
\text { Admission } \\
J \\
\text { Antivenom } \\
\end{array}$ & $\begin{array}{l}\text { Time Interval } \\
\text { Bite-Death }\end{array}$ & $\begin{array}{l}\text { Snake } \\
\text { Family }\end{array}$ & $\begin{array}{c}\text { Bite Area } \\
\text { Site } \\
\text { Macroscopical Findings }\end{array}$ & $\begin{array}{c}\text { Bite Area } \\
\text { Histological Findings }\end{array}$ & $\begin{array}{c}\text { Autopsy } \\
\text { Macroscopical Findings }\end{array}$ & $\begin{array}{c}\text { Autopsy } \\
\text { Histological Pattern }\end{array}$ \\
\hline $\begin{array}{l}\text { Norris RL et al., } \\
2009 \text { [29] }\end{array}$ & USA & $38 / \mathrm{M}$ & No & No; No & $2 \mathrm{~h}$ & $\begin{array}{l}\text { Coral snake (Micrurus } \\
\text { fulvius) Elapidae }\end{array}$ & $\begin{array}{l}\text { Right Hand; Left Arm. } \\
\text { rresh } 6 \text { mm linear } \\
\text { abrasion and multiple } \\
\text { punctuate abrasions in a } \\
\text { small group. }\end{array}$ & n.a. & L: congestion. & Unremarkable. \\
\hline $\begin{array}{l}\text { Okamoto O et al., } \\
2017 \text { [30] }\end{array}$ & Japan & $84 / \mathrm{F}$ & Yes & Yes; Yes & $35 \mathrm{~d}$ & $\begin{array}{l}\text { Mamushi (Gloydius } \\
\text { Blomhoffii) Viperidae }\end{array}$ & Right elbow. & n.a. & $\begin{array}{l}\text { Li: acute necrosis. } \\
\text { I: extensive patchy } \\
\text { necrosis at the small } \\
\text { intestine; multiple ulcers } \\
\text { and bleeding at the } \\
\text { ascending colon. } \\
\text { K: cortical necrosis. }\end{array}$ & $\begin{array}{l}\text { Li: necrosis around the central vein } \\
\text { I: necrosis extended to the muscle } \\
\text { layer. } \\
\text { K: glomerular necrosis. }\end{array}$ \\
\hline $\begin{array}{l}\text { Paulis MG et al., } \\
2016[31]\end{array}$ & Egypt & 9/F & No & No; Yes & n.a. & $\begin{array}{c}\text { Egyptian Cobra (Naja } \\
\text { Haje) Elapidae }\end{array}$ & $\begin{array}{l}\text { Right leg. } \\
\text { Five pairs of fang marks } \\
\text { with almost fixed } \\
\text { distance between the } \\
\text { paired ones, all } \\
\text { surrounded by erythema } \\
\text { and little swelling. }\end{array}$ & n.a. & $\begin{array}{l}\text { Internal organ } \\
\text { congestion. }\end{array}$ & n.a. \\
\hline $\begin{array}{l}\text { Paulis MG et al., } \\
2016[31]\end{array}$ & Egypt & 6/F & No & No; No. & n.a. & $\begin{array}{l}\text { Egyptian Cobra (Naja } \\
\text { Haje) Elapidae }\end{array}$ & $\begin{array}{l}\text { Left leg. } \\
\text { Two pairs of fang marks, } \\
\text { each pair was } \\
\text { symmetrical, all } \\
\text { surrounded by erythema } \\
\text { and little swelling. }\end{array}$ & n.a. & $\begin{array}{l}\text { Internal organ } \\
\text { congestion. }\end{array}$ & n.a. \\
\hline $\begin{array}{l}\text { Paulis MG et al., } \\
2016[31]\end{array}$ & Egypt & $4 / \mathrm{F}$ & No & No; No. & n.a. & $\begin{array}{l}\text { Egyptian Cobra } \\
\text { (Naja Haje) Elapidae }\end{array}$ & $\begin{array}{l}\text { Right leg. } \\
\text { Multiple fang marks } \\
\text { close to each other } \\
\text { surrounded by } \\
\text { erythema. }\end{array}$ & n.a. & $\begin{array}{l}\text { Internal organ } \\
\text { congestion. }\end{array}$ & n.a. \\
\hline $\begin{array}{l}\text { Pramod Kumar } \\
\text { GN et al, 2013 [32] }\end{array}$ & India & $68 / \mathrm{M}$ & n.a. & Yes; n.a. & $4 \mathrm{~d}$ & n.a. & $\begin{array}{l}\text { Right thigh. } \\
\text { Bluish black } \\
\text { discoloration of the } \\
\text { outer aspect of the rirght } \\
\text { thigh and of the right leg. } \\
\text { Multiple blisters and } \\
\text { ulcerative necrosis. } \\
\text { Extravasation of blood in } \\
\text { the subcutaneous plane. }\end{array}$ & n.a. & $\begin{array}{l}\text { Unremarkable } \\
\text { (coexistence of gastric } \\
\text { and kidney cancers). }\end{array}$ & n.a. \\
\hline Quain, $1852[33]$ & $\begin{array}{l}\text { England } \\
\text { (captivity) }\end{array}$ & $30 / \mathrm{M}$ & Yes & Yes: No. & $1 \mathrm{~h} 30 \mathrm{~min}$ & $\begin{array}{l}\text { Hooded Snake - Cobra } \\
\text { de Capello (Naja } \\
\text { tripudians) Elapidae }\end{array}$ & $\begin{array}{c}\text { Face. } \\
\text { Three puncture marks } \\
\text { surrounded by an } \\
\text { ecchymotic area. }\end{array}$ & n.a. & $\begin{array}{c}\text { Congestion of all } \\
\text { internal organs, } \\
\text { particularly of the } \\
\text { spleen. }\end{array}$ & n.a. \\
\hline $\begin{array}{l}\text { Rathnayaka } \\
\text { RMMKN et al., } \\
2017 \text { [34] }\end{array}$ & Sri Lanka & $53 / \mathrm{M}$ & n.a. & Yes; Yes & $26 \mathrm{~d}$ & $\begin{array}{l}\text { Russell's viper (Daboia } \\
\text { russelii) Viperidae }\end{array}$ & $\begin{array}{l}\text { Right Leg. } \\
\text { Swollen area. }\end{array}$ & n.a. & $\begin{array}{l}\text { B: infarcted area of } \\
\text { parietal lobes of the } \\
\text { brain. } \\
\text { K: reduced } \\
\text { cortico-medullary } \\
\text { demarcation in both } \\
\text { kidneys and petechial } \\
\text { hemorrhages. }\end{array}$ & n.a. \\
\hline
\end{tabular}


Table 1. Cont

\begin{tabular}{|c|c|c|c|c|c|c|c|c|c|c|}
\hline References & Country & $\begin{array}{c}\text { Victim } \\
\text { Age/Sex }\end{array}$ & $\begin{array}{l}\text { Work- } \\
\text { Related }\end{array}$ & $\begin{array}{c}\text { Hospital } \\
\text { Admission } \\
J \\
\text { Antivenom } \\
\end{array}$ & $\begin{array}{l}\text { Time Interval } \\
\text { Bite-Death }\end{array}$ & $\begin{array}{l}\text { Snake } \\
\text { Family }\end{array}$ & $\begin{array}{c}\text { Bite Area } \\
\text { Site } \\
\text { Macroscopical Findings }\end{array}$ & $\begin{array}{c}\text { Bite Area } \\
\text { Histological Findings }\end{array}$ & $\begin{array}{l}\text { Autopsy } \\
\text { Macroscopical Findings }\end{array}$ & $\begin{array}{c}\text { Autopsy } \\
\text { Histological Pattern }\end{array}$ \\
\hline $\begin{array}{l}\text { Sarkar N et al., } \\
\quad 2018[3]\end{array}$ & India & $27 / \mathrm{M}$ & No & No; No. & almost $6 \mathrm{~h}$ & $\begin{array}{l}\text { Banded Krait (Bungarus } \\
\text { faciatus) Elapidae }\end{array}$ & $\begin{array}{c}\text { Hand. } \\
\text { Two pin-point } \\
\text { punctured wounds, } \\
\text { subcutaneous deep, } \\
\text { placed } 8 \mathrm{~mm} \text { apart. }\end{array}$ & n.a. & $\begin{array}{l}\text { Internal organ } \\
\text { congestion } \\
\text { K: bilateral } \\
\text { cortico-medullary } \\
\text { hemorrhage and } \\
\text { congestion. } \\
\end{array}$ & $\begin{array}{l}\text { K: interstitial hemorrhage; } \\
\text { inflammatory cell infiltration } \\
\text { and swelling. }\end{array}$ \\
\hline $\begin{array}{l}\text { Sarkar N et al., } \\
\quad 2018 \text { [3] }\end{array}$ & India & 26/F & No & No; No & almost $6 \mathrm{~h}$ & $\begin{array}{l}\text { Banded Krait (Bungarus } \\
\text { faciatus) Elapidae }\end{array}$ & $\begin{array}{l}\text { Right shoulder. } \\
\text { Two pin-point } \\
\text { punctured wounds. }\end{array}$ & n.a. & $\begin{array}{l}\text { Internal organ } \\
\text { congestion } \\
\text { Kn bilateral } \\
\text { cortico-medullary } \\
\text { hemorrhage and } \\
\text { congestion. }\end{array}$ & $\begin{array}{l}\text { K: interstitial hemorrhage and } \\
\text { inflammatory cell infiltration. }\end{array}$ \\
\hline $\begin{array}{l}\text { Sarkar N et al., } \\
2018[3]\end{array}$ & India & $4 / \mathrm{F}$ & No & No; No & almost $6 \mathrm{~h}$ & $\begin{array}{l}\text { Banded Krait (Bungarus } \\
\text { faciatus) Elapidae }\end{array}$ & Right foot. & n.a. & $\begin{array}{l}\text { Internal organ } \\
\text { congestion. } \\
\text { K: bilateral } \\
\text { cortico-medullary } \\
\text { hemorrhage and } \\
\text { congestion. }\end{array}$ & $\begin{array}{l}\text { K: increased cytoplasmatic } \\
\text { eosinophilia and occasional surface } \\
\text { blebbing of the renal tubular } \\
\text { epithelia cell (early } \\
\text { ischemic injury). }\end{array}$ \\
\hline $\begin{array}{c}\text { Silva A et al, } 2013 \\
\text { [35] }\end{array}$ & Sri Lanka & $19 / \mathrm{M}$ & No & Yes; Yes & $1 \mathrm{~d} 22 \mathrm{~h}$ & $\begin{array}{l}\text { Indian Krait (Bungarus } \\
\text { caeruleus) Elapidae }\end{array}$ & n.a. & n.a. & $\begin{array}{l}\text { H, L, K: Petechial } \\
\text { hemorrhages and } \\
\text { edema. }\end{array}$ & n.a. \\
\hline $\begin{array}{l}\text { Silva de Oliveira S } \\
\text { et al., 2017 [36] }\end{array}$ & Brazil & 59/F & No & Yes; Yes & $2 \mathrm{~d}$ & $\begin{array}{l}\text { Jaracaca do Norte } \\
\text { (Bothrops atrox) } \\
\text { Viperidae }\end{array}$ & $\begin{array}{l}\text { Right foot. } \\
\text { Ecchymotic and swollen } \\
\text { area. }\end{array}$ & n.a. & $\begin{array}{l}\text { B: Subarachnoid and } \\
\text { intraparenchymal } \\
\text { hemorrhage. }\end{array}$ & n.a. \\
\hline $\begin{array}{l}\text { Soe S et al.,1993 } \\
{[37]}\end{array}$ & Myanmar & $13 / \mathrm{M}$ & n.a. & n.a.; n.a. & n.a. & $\begin{array}{l}\text { Russell's Viper } \\
\text { (Vipera Russelli) } \\
\quad \text { Viperidae }\end{array}$ & n.a. & n.a. & n.a. & $\begin{array}{l}\text { K: Glomerular changes: } \\
\text { endothelial swelling, increased } \\
\text { cells, degeneration and necrosis of } \\
\text { the tubules, and fibrin deposition. }\end{array}$ \\
\hline $\begin{array}{l}\text { Soe S et al.,1993 } \\
{[37]}\end{array}$ & Myanmar & $15 / \mathrm{F}$ & n.a. & n.a.; n.a. & n.a. & $\begin{array}{l}\text { Russell's Viper } \\
\text { (Vipera Russelli) } \\
\text { Viperidae }\end{array}$ & n.a. & n.a. & n.a. & $\begin{array}{l}\text { K: Glomerular changes: } \\
\text { endothlial swelling, increased } \\
\text { cells; degeneneration and necrosis of } \\
\text { the tubules, and fibrin deposition. }\end{array}$ \\
\hline $\begin{array}{l}\text { Soe S et al.,1993 } \\
{[37]}\end{array}$ & Myanmar & $15 / \mathrm{F}$ & n.a. & n.a.; n.a. & n.a. & $\begin{array}{l}\text { Russell's Viper } \\
\text { (Vipera Russelli) } \\
\quad \text { Viperidae }\end{array}$ & n.a. & n.a. & n.a. & $\begin{array}{l}\text { K: Glomerular changes: } \\
\text { endothelial swelling, increased } \\
\text { cells, degeneration and necrosis of } \\
\text { the tubules, and fibrin deposition. }\end{array}$ \\
\hline $\begin{array}{l}\text { Soe S et al.,1993 } \\
{[37]}\end{array}$ & Myanmar & $49 / \mathrm{M}$ & n.a. & n.a.; n.a. & n.a. & $\begin{array}{l}\text { Russell's Viper } \\
\text { (Vipera Russelli) } \\
\text { Viperidae }\end{array}$ & n.a. & n.a. & n.a. & $\begin{array}{l}\text { K: Glomerular changes: } \\
\text { endothelial swelling, increased } \\
\text { cells; degenenation and necrosis of } \\
\text { the tubules, and fibrin deposition }\end{array}$ \\
\hline $\begin{array}{l}\text { Sutherland SK, } \\
1992[38]\end{array}$ & Australia & 16/F & No & Yes; n.a. & $1 \mathrm{~h}$ & $\begin{array}{c}\text { Brown snake } \\
\text { (Pseudonaja Textilis) } \\
\text { Elapidae }\end{array}$ & $\begin{array}{c}\text { Right heel. } \\
\text { Fang marks } 5 \mathrm{~mm} \text { apart. }\end{array}$ & n.a. & Unremarkable. & n.a. \\
\hline $\begin{array}{c}\text { Sutherland SK, } \\
1992[38]\end{array}$ & Australia & $27 / \mathrm{F}$ & n.a. & Yes; n.a. & $1 \mathrm{~h} 30 \mathrm{~min}$ & Brown snake Elapidae & n.a. & n.a. & Unremarkable. & n.a. \\
\hline $\begin{array}{l}\text { Sutherland SK, } \\
1992[38]\end{array}$ & Australia & $61 / \mathrm{F}$ & NO & Yes; No & $1 \mathrm{~h} 30 \mathrm{~min}$ & Brown snake Elapidae & Right ankle. & n.a. & $\begin{array}{l}\text { Small retroperitoneal } \\
\text { hemorrhage. }\end{array}$ & n.a. \\
\hline $\begin{array}{c}\text { Sutherland SK, } \\
1992[38]\end{array}$ & Australia & $51 / \mathrm{M}$ & n.a. & Yes; Yes & $1 \mathrm{~h}$ & $\begin{array}{l}\text { Taipan } \\
\text { Elapidae }\end{array}$ & n.a. & n.a. & Unremarkable. & n.a. \\
\hline $\begin{array}{l}\text { Sutherland SK, } \\
1992[38]\end{array}$ & Australia & $2 / \mathrm{F}$ & No & No; No & n.a. & Brown snake Elapidae & $\begin{array}{l}\text { Left leg. } \\
\text { Two minute puncture } \\
\text { wounds. }\end{array}$ & n.a. & $\begin{array}{l}\text { L: congestion and edema } \\
\text { with numerous petechial } \\
\text { hemorrhages on the } \\
\text { visceral pleurae. }\end{array}$ & n.a. \\
\hline
\end{tabular}


Table 1. Cont

\begin{tabular}{|c|c|c|c|c|c|c|c|c|c|c|}
\hline References & Country & $\begin{array}{c}\text { Victim } \\
\text { Age/Sex }\end{array}$ & $\begin{array}{l}\text { Work- } \\
\text { Related }\end{array}$ & $\begin{array}{c}\text { Hospital } \\
\text { Admission } \\
J \\
\text { Antivenom } \\
\end{array}$ & $\begin{array}{l}\text { Time Interval } \\
\text { Bite-Death }\end{array}$ & $\begin{array}{l}\text { Snake } \\
\text { Family }\end{array}$ & $\begin{array}{c}\text { Bite Area } \\
\text { Site } \\
\text { Macroscopical Findings } \\
\end{array}$ & $\begin{array}{l}\text { Bite Area } \\
\text { Histological Findings }\end{array}$ & $\begin{array}{l}\text { Autopsy } \\
\text { Macroscopical Findings }\end{array}$ & $\begin{array}{c}\text { Autopsy } \\
\text { Histological Pattern }\end{array}$ \\
\hline $\begin{array}{c}\text { Sutherland SK, } \\
1992[38]\end{array}$ & Australia & $42 / \mathrm{M}$ & No & Yes; Yes & $5 \mathrm{~h} 30 \mathrm{~min}$ & $\begin{array}{l}\text { Taipan } \\
\text { Elapidae }\end{array}$ & n.a. & n.a. & Unremarkable. & n.a. \\
\hline $\begin{array}{c}\text { Sutherland SK, } \\
1992[38]\end{array}$ & Australia & $60 / \mathrm{M}$ & Yes & Yes; Yes & $25 \mathrm{~min}$ & Brown snake Elapidae & Right Foot. & n.a. & Unremarkable. & n.a. \\
\hline $\begin{array}{l}\text { Sutherland SK, } \\
1992[38]\end{array}$ & Australia & $35 / \mathrm{M}$ & No & No; No & $35 \mathrm{~min}$ & $\begin{array}{c}\text { Brown snake } \\
\text { (Pseudonaja Textilis) } \\
\text { Elapidae }\end{array}$ & Right hand. & n.a. & $\begin{array}{l}\text { H: Coronary arteries } \\
\text { disease. } \\
\text { Li: cirrhosis. }\end{array}$ & n.a. \\
\hline $\begin{array}{l}\text { Sutherland SK, } \\
\quad 1992[38]\end{array}$ & Australia & $48 / \mathrm{F}$ & No & No; No & $30 \mathrm{~min}$ & n.a. & $\begin{array}{l}\text { Right Foot. } \\
\text { Two tiny puncture } \\
\text { wounds. }\end{array}$ & n.a. & n.a. & $\begin{array}{l}\text { Quantitites of venom in urine that } \\
\text { reacted to tiger snake venom. }\end{array}$ \\
\hline $\begin{array}{l}\text { Than-Than et al., } \\
1989[39]\end{array}$ & Myanmar & $19 / \mathrm{M}$ & Yes & Yes (at the end); Yes & $14 \mathrm{~h}$ & $\begin{array}{c}\text { Russell's Viper } \\
\text { (Vipera Russellii } \\
\text { Siamensis) Viperidae }\end{array}$ & $\begin{array}{c}\text { Ankle. } \\
\text { bruising area. }\end{array}$ & n.a. & $\begin{array}{l}\text { L: petechial } \\
\text { hemorrhages. }\end{array}$ & 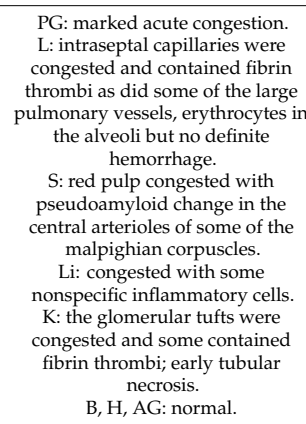 \\
\hline $\begin{array}{l}\text { Than-Than et al., } \\
1989[39]\end{array}$ & Myanmar & $17 / \mathrm{M}$ & Yes & Yes (at the end); Yes & $1 \mathrm{~d} 12 \mathrm{~h}$ & $\begin{array}{c}\text { Russell's Viper } \\
\text { (Vipera Russelli } \\
\text { Siamensis) Viperidae }\end{array}$ & Foot. & $\begin{array}{c}\text { Hemorrhage, necrosis, } \\
\text { and the presence of } \\
\text { fibrin thrombi in mall } \\
\text { vessels. Subcutaneous } \\
\text { fibrofatty tissue heavily } \\
\text { iffiltrated by a mixture } \\
\text { of PMNs, leucocytes, } \\
\text { lymphocytes, and } \\
\text { eosinophils. Dense } \\
\text { acidophilic } \\
\text { homogeneous fibrin-like } \\
\text { material in the } \\
\text { surrounding blood } \\
\text { vessels. } \\
\text { vesse }\end{array}$ & $\begin{array}{l}\text { Serous fluid in the } \\
\text { pleural and peritoneal } \\
\text { cavities. } \\
\text { B: edema. } \\
\text { PGG AG: diffusely } \\
\text { hemorrhagic. } \\
\text { H, L: petechial } \\
\text { hemorrhages. } \\
\text { K: demarcation between } \\
\text { dark red medulla and } \\
\text { pale cortex. }\end{array}$ & 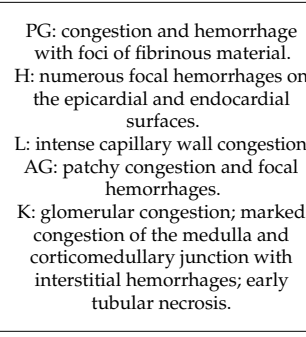 \\
\hline $\begin{array}{l}\text { Than-Than et al., } \\
1989[39]\end{array}$ & Myanmar & 15/F & Yes & Yes; Yes & $2 \mathrm{~d} 4 \mathrm{~h}$ & $\begin{array}{c}\text { Russell's Viper } \\
\text { (Vipera Russelli } \\
\text { Siamensis) Viperidae }\end{array}$ & Foot. & n.a. & $\begin{array}{l}\text { B: edematous and } \\
\text { congested. Large blood } \\
\text { clot }(5 \times 12 \mathrm{~mm} \text { ) in the } \\
\text { pitiutary foss. } \\
\text { PG: dark grey in color } \\
\text { and hemorrhagic } \\
\text { parenchymag. } \\
\text { P, H, St: subserosal } \\
\text { hemorrhage. } \\
\text { AG: hemorragic. } \\
\text { K: Blood clots in the } \\
\text { pelvises. Contrasting red } \\
\text { medulla and pale cortex. }\end{array}$ & 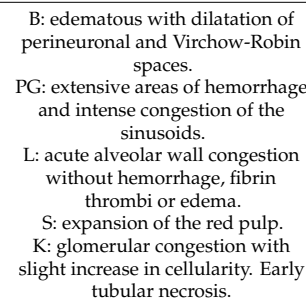 \\
\hline
\end{tabular}


Table 1. Cont.

\begin{tabular}{|c|c|c|c|c|c|c|c|c|c|c|}
\hline References & Country & $\begin{array}{c}\text { Victim } \\
\text { Age/Sex }\end{array}$ & $\begin{array}{l}\text { Work- } \\
\text { Related }\end{array}$ & $\begin{array}{l}\text { Hospital } \\
\text { Admission } \\
\text { Intivenom }\end{array}$ & $\begin{array}{l}\text { Time Interval } \\
\text { Bite-Death }\end{array}$ & $\begin{array}{l}\text { Snake } \\
\text { Family }\end{array}$ & $\begin{array}{c}\text { Bite Area } \\
\text { Site } \\
\text { Macroscopical Findings }\end{array}$ & $\begin{array}{l}\text { Bite Area } \\
\text { Histological Findings }\end{array}$ & $\begin{array}{c}\text { Autopsy } \\
\text { Macroscopical Findings }\end{array}$ & $\begin{array}{c}\text { Autopsy } \\
\text { Histological Pattern }\end{array}$ \\
\hline $\begin{array}{l}\text { Tibballs J et al., } \\
1991[40]\end{array}$ & Australia & $11 / \mathrm{M}$ & No & Yes; Yes & Less than $1 \mathrm{~d}$ & $\begin{array}{l}\text { Tiger snake (Notechis } \\
\text { scutatus) Elapidae }\end{array}$ & Left wrist. & n.a. & $\begin{array}{l}\text { B: multiple intracerebral } \\
\text { hemorrhages and } \\
\text { subarachnood collections } \\
\text { of blood. }\end{array}$ & n.a. \\
\hline $\begin{array}{l}\text { Tilbury CR et al., } \\
2016[41]\end{array}$ & Congo & $46 / \mathrm{M}$ & Yes & Yes; Yes & $2 \mathrm{~h} 20 \mathrm{~min}$ & $\begin{array}{c}\text { Burrowing Asps } \\
\text { (Atractaspis corpulenta) } \\
\text { Lamprophiiddae }\end{array}$ & $\begin{array}{l}\text { Left hand. } \\
\text { Punctuation-shaped } \\
\text { wound (!), } 2.5 \mathrm{~mm} \text { i in } \\
\text { length surrounded by a } \\
\text { non-specified rash. }\end{array}$ & $\begin{array}{l}\text { Disruption of the } \\
\text { epidermal and dermal } \\
\text { tissue layerss, with } \\
\text { associated fresh } \\
\text { hemorrhage. }\end{array}$ & $\begin{array}{l}\text { Diffuse congestion of the } \\
\text { organs. } \\
\text { H: flabby. } \\
\text { L: edematous and } \\
\text { congested. }\end{array}$ & $\begin{array}{l}\text { H: acute ischemic changes, foci of } \\
\text { contraction band necrosis with no } \\
\text { accompanying inflammatory } \\
\text { response. } \\
\text { L: prominent pulmonary edema } \\
\text { and severe pulmonary congestion }\end{array}$ \\
\hline $\begin{array}{l}\text { Tranca S et al., } \\
2016 \text { [42] }\end{array}$ & Romania & $56 / \mathrm{M}$ & n.a. & Yes; Yes & n.a. & Vipera Berus Viperidae & $\begin{array}{l}\text { Right tight. } \\
\text { Two puncture wounds } \\
\text { surrounded by a } \\
\text { purpuric area. }\end{array}$ & $\begin{array}{l}\text { Diffuse epidermal } \\
\text { necrosis associated with } \\
\text { micro-hemorrhages and } \\
\text { micro-thrombosis in the } \\
\text { dermis and hypodermis. }\end{array}$ & H, L: edema. & $\begin{array}{l}\text { B: edema and hemorrhagic lesions } \\
\text { in the leptomenninges. } \\
\text { H: myocardial fibrosis, epicardial } \\
\text { and interstitial hemorrhages. } \\
\text { L: atelectasia and pulmanary } \\
\text { hemorrhagic edema. } \\
\text { Li: diffuse hepatic necrosis. } \\
\text { I: enteral necrosis and acute } \\
\text { mucosal hemorrhages. } \\
\text { K: acute tubular necrosis. }\end{array}$ \\
\hline $\begin{array}{l}\text { Varagunam T et } \\
\text { al., } 1970[43]\end{array}$ & Sri Lanka & $30 / \mathrm{M}$ & Yes & Yes; No & $11 \mathrm{~d}$ & $\begin{array}{l}\text { Pit-Viper (Agkistrodom } \\
\text { hypnale) Viperidae }\end{array}$ & $\begin{array}{l}\text { Left hand. } \\
\text { Scars of two fang marks. }\end{array}$ & n.a. & $\begin{array}{l}\text { K: larger, external } \\
\text { surface was smooth with } \\
\text { many areas of necrosis } \\
\text { or hemorrhage; necrotic } \\
\text { areas confined to the } \\
\text { cortices; the medullae } \\
\text { were congested. } \\
\text { H, L, Li, S, I, AG: normal. }\end{array}$ & $\begin{array}{l}\text { Li: early fatty changes. } \\
\text { K: irregular areas of coagulative } \\
\text { necrosis in the cortices; both } \\
\text { glomeruli and tubules were } \\
\text { involved in the necrotic process; } \\
\text { Necrosis in many intralobular } \\
\text { arteries and arterioles. }\end{array}$ \\
\hline $\begin{array}{l}\text { Wankhede AG, } \\
2004[44]\end{array}$ & India & $25 / \mathrm{M}$ & Yes & Yes, n.a. & $1 \mathrm{~d}$ & $\begin{array}{l}\text { Russell's Viper (Vipera } \\
\text { Russelli Siamensis) } \\
\text { Viperidae }\end{array}$ & $\begin{array}{l}\text { Right ankle. } \\
\text { Two puncture wounds of } \\
\text { diameter } 1.5 \mathrm{~mm} \text { each } \\
\text { and } 2.3 \mathrm{~cm} \text { apart and } \\
\text { puncture lacerated } \\
\text { wounds one } 7 \mathrm{~mm} \text { below } \\
\text { the lateral puncture } \\
\text { wound, } 1.5 \mathrm{~cm} \text { in length, } \\
\text { and the second } 1.6 \mathrm{~cm} \\
\text { below the medial } \\
\text { puncture wound } 6 \mathrm{~mm} \\
\text { in length. }\end{array}$ & n.a. & $\begin{array}{l}\text { B: petechial } \\
\text { hemorrhages, congestion } \\
\text { and edema. } \\
\text { L: congestion and } \\
\text { edema. }\end{array}$ & Unremarkable. \\
\hline $\begin{array}{l}\text { Warrell DA et al., } \\
\quad 1976[45]\end{array}$ & Nigeria & $10 / \mathrm{M}$ & No & No; No & $2 \mathrm{~h}$ & $\begin{array}{l}\text { Egyptian Cobra (Naja } \\
\text { Haje) Elapidae }\end{array}$ & $\begin{array}{l}\text { Right hand. } \\
\text { Two fang punctures } 2 \mathrm{~cm} \\
\text { apart, surrounded by a } \\
\text { swollen area. }\end{array}$ & n.a. & Unremarkable. & B, H: normal. \\
\hline
\end{tabular}

AG: adrenal glands; ARDS: acute respiratory distress syndrome; B: brain; DIC: disseminated intravascular coagulation; H: heart; I: intestine; K: kidneys; L: lun
M: muscle; $n$. a: not available; P: pancreas; PG: pituitary gland; PMNs: polymorphonuclear leukocytes; RA: right atrium; S: spleen; St: stomach; T: thymus. 


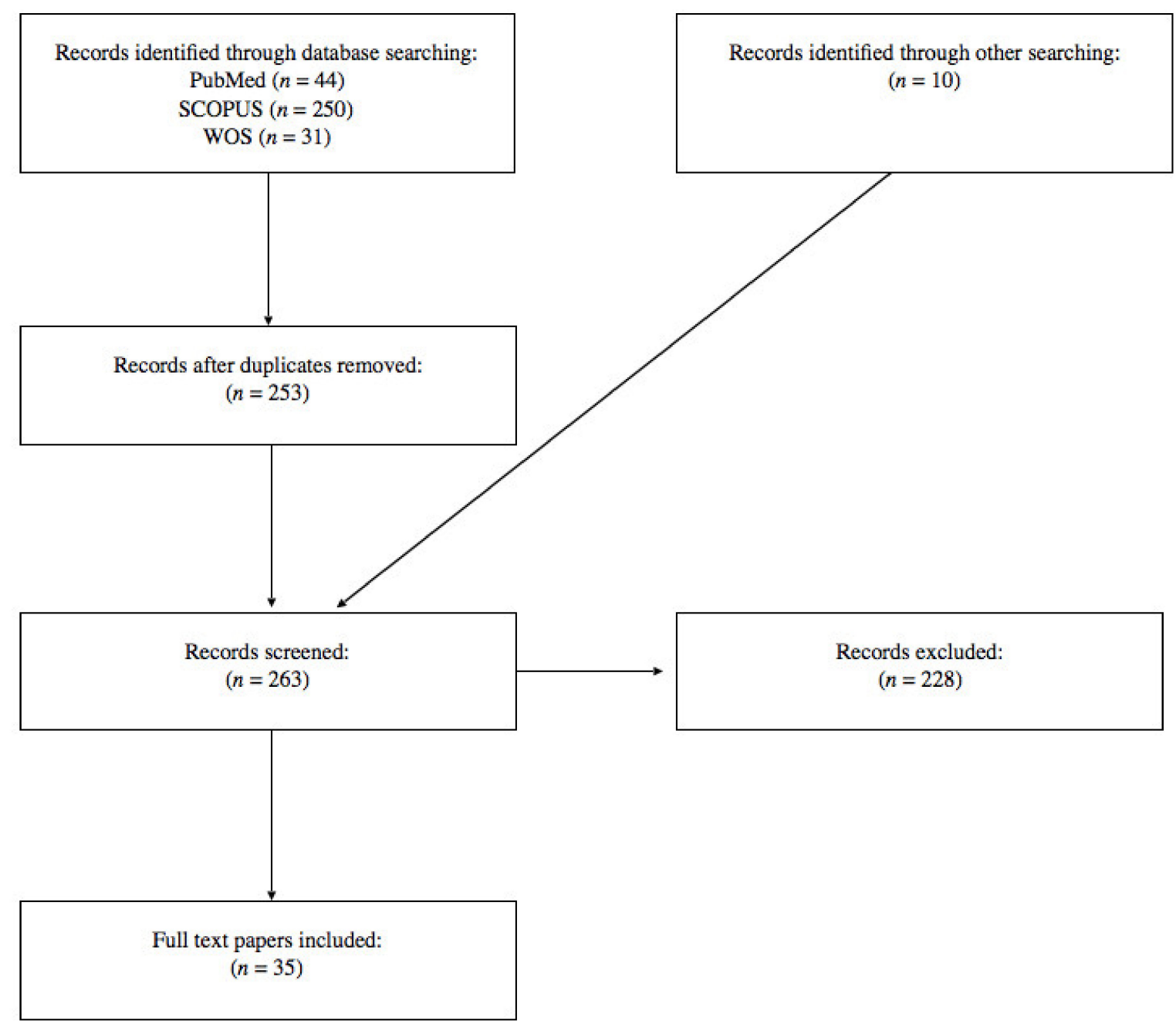

Figure 1. Flowchart depicting the choice of the studies.

\subsection{Epidemiological Findings}

The most common families of snakes involved in fatal SBE were Viperidae ( $n=28-50.000 \%$ ) followed soon after by Elapidae $(n=23-41.071 \%)$ and Lamprophiidae $(n=1-1.786 \%)$. In four cases $(7.143 \%)$ no additional information about the snake was available. Most of the fatal cases of SBE reported can be classified into category $1(n=28)$ according to the WHO Guidelines on production, control, and regulation of snake antivenom immunoglobulins [8]. Just in two cases [22,33], the snake was not included in that list and in both cases, the snake was kept in captivity far from his habitat.

Most of the SBE victims were males $(n=35$ cases; $62.500 \%)$ with a male/female ratio $(\mathrm{M} / \mathrm{F})$ of 1.67 . Male victims were older than females. The median age in years for males was 40.77 and for females 28.71. The overlapping mean age was 36.25 years.

The body part most frequently bitten by snakes was the lower limb in 31 cases ( $55.357 \%)$, the upper limbs in 15 cases $(26.786 \%)$, in one case the face $(1.786 \%)$, and in one other case the genitalia $(1.786 \%)$. Unfortunately, in eight cases (14.286\%) the site of the snakebite was not reported. The vast majority of the SBE was accidental, but in one case study involving three victims, the manner of death was assessed as homicide [31].

The largest number of victims $(n=12)$ is reported from Australia, followed by Myanmar and India ( $n=7$ each), Brazil and Sri-Lanka $(n=6$ each). However, all continents in the world are represented: $41.071 \%$ of victims were from Asia $(n=23), 21.429 \%$ from Oceania $(n=12), 19.643 \%$ from America (Northern, Central, and Southern; $n=11), 14.286 \%$ from Africa $(n=8)$, and 3.571\% from Europe $(n=2)$. 
It is worth mentioning that 16 out of 56 cases were related to work activities. Most of the cases occurred in a rural context (countryside, paddies, etc.).

Regarding clinical history, the time interval between snakebites and death ranges from $25 \mathrm{~min}$ up to 35 days. Deaths occurred mostly after $24 \mathrm{~h}$ for 22 cases $(39.286 \%)$ and before $6 \mathrm{~h}$ in 19 cases $(33.929 \%)$. Most of the victims, 40 out of 56 cases (71.429\%), were hospitalized and the specific antivenom was administered to 26 individuals. In a few cases, the victim was at first treated by a local healer or with traditional remedies $[15,20,28,43]$.

\subsection{Post Mortem Examination Findings}

Most of the bite marks (19 out of 56 cases) are described as two puncture wounds from the animal's fangs. Just a single puncture wound has been found in three cases, sometimes scratched or lacerated by the movement of the victim and/or his clothing deviating the fangs [44]. Additional wounds caused by other teeth or additional bites have been also described [29,31,43]. In two cases there were three fang marks [24,33]. Wankhede (2004) [44] described an unusual case in which four puncture wounds due to a single snakebite were found. In this strange case, the presence of the fang marks was consistent with a snakehead bigger in size than usual where the old fangs were still present, along with two new fangs.

In this regard, it is worth mentioning that for the identification of the offending snake the number of puncture wounds must be related to the number of teeth, which is unfortunately not the same in all kinds of snakes. Different species of snakes can have different anatomical conformation of the teeth and fangs. Fangs are sharp, long, hollow, or grooved teeth that are connected to a small sack full of venom in the snake's head behind its eyes but not all snakes have fangs. Most snakes have six rows of teeth in total: one row on each lower jaw and two rows on each maxillary and palatine or pterygoid bones of the upper jaw [46]. It is also important to highlight that the victim's clothes or gloves can represent an obstacle to the penetration of a fang [41].

With regard to the morphology of the fangs, sometimes the bite injuries are also described as linear or curvilinear stripes [15] fresh linear or multiple punctate abrasion [29]. The morphology of this kind of injury is quite similar to those produced by sharp force [47]. Snakebites in the multiple punctate abrasion pattern are often the result of the typical chewing activity of the coral snake. Instead, in most of the pit viper bites one or more fang marks are usually found [48] due to the stabbing motion of this snake specie's bite [29]. Although important for the identification of the offending snake, only in very few cases $(n=8)[3,12,20,22,24,37,44,45]$, the distance between the fang marks is reported. At the external examination, the area surrounding the bite mark shows dermal alterations represented mostly by signs of flogosis such as edema, cellulitis, and erythema. Bruises related to the snake bites are also reported due to the bleeding into the skin. It is strongly recommended to check the signs of the vital reaction of the bite mark and to take samples from bitten tissue. Subcutaneous hemorrhages are pretty common at the puncture skin site along with skin necrosis caused by the snake venom components. Only very few cases report additional dermal effects of the venom such as multiple blisters [32], a rash [41], and a purpuric area due to microthrombosis of the skin [42]. The main histological findings are subcutaneous hemorrhage, rhabdomyolysis, and necrosis of the dermis. Autopsy findings are mostly non-specific general pathologic changes and consistent with a rapid death such as multi-visceral congestion or edema and petechial hemorrhages. In the brain, other than congestion and edema, petechial hemorrhages from pin-point size to large hematoma in the white matter have been reported in 13 cases out of 56 in total $(23.214 \%)[14-16,18,20,23-25,27,39,44]$. Microscopically, signs of ischemic and hemorrhagic encephalopathy have been found in six cases out of 56 [14,24,26,28,34,36] and extensive perivascular demyelination in a single case [21] Myocardial hemorrhages have been reported in seven cases out of $56(12.500 \%)[12,14,16,27,35,39]$ and alterations consistent with myocardial infarction in a single case [14]. Signs of rapid failure of the left ventricle were mostly represented by pulmonary edema and congestion. Additional histological findings are hyaline membranes due to diffuse alveolar damage (DAD) in three cases [16,24,25] and widespread microthrombosis consistent with a disseminated intravascular coagulation (DIC) in four cases $(7.142 \%)[12,19,20,39]$. Renal injuries other than vascular 
congestion were mainly represented by tubular and/or glomerular necrosis found in 13 cases out of 56 in total $(23.214 \%)[11,15,19,21,24,26,37,39,42]$. Cortical and glomerular necrosis has been reported in one single case [30] and widespread ischemic necrosis in a different one [43]. Microthrombosis of the small inter-lobular vessels was found in a single case. Additional renal alterations are represented by acute interstitial nephritis (AIN) in three cases [3]. Intestinal injuries are reported in only three cases, but no better described [20,30,42]. Pituitary hemorrhage/necrosis and adrenal gland hemorrhage were present in another three cases [39].

\section{Discussion}

SBE is an acute medical emergency and a major public health problem that kills thousands of people each year, particularly in rural territories [49]. Most of the case studies considered in the present retrospective study were coming from tropical countries such as Brazil, Congo, India, Martinique, Myanmar, Nigeria, and Sri Lanka. The majority of fatal SBE involved snakes belonging to the Elapidae family (e.g., kraits-Bungarus spp., cobras-Naja spp., taipans-Oxyuranus spp., coral snakes-Micrurus spp., death adders-Acanthophis spp., and tiger snakes-Notechis spp.) and to the Viperidae family (e.g., rattlesnakes-Crotalus spp., Russell's viper-Daboia russelii). According to the geographical latitudes, the species of snakes causing the largest numbers of bites and fatalities are Echis sp. (saw-scaled vipers) in Northern Africa, Bothrops asper and B. atrox (lance-headed pit vipers) in Central and South America, and Naja sp. (cobras) and Bungarus sp. (kraits) in Asia [50]. SBE is both a method of hunting and a means of protection [51]. Snake venom is produced by specialized venom glands located in the upper jaw and associated with fangs, which are specialized long teeth with a superficial groove, along which the venom runs. Sometimes the fangs can also have an enclosed canal, down which the venom flows inside the tooth [52]. The severity of SBE depends on several factors which include bite localization, snake's size, condition of glands and teeth, bite angle and bite duration, microflora of the snake's mouth and victim's skin, age and weight of the victim, health status, victim's activity after a bite [53-55].

However, the main deadly factor is represented by the components of the venom. Snake venoms are mixtures of protein families, and each of these families contains many different toxins or toxin isoforms [56]. A very recent proteomic study on the venom of the viperid genus indicates the presence of proteins belonging to at least 12 families, with a predominance of phospholipases A2 (PLA2s) (54\%) and metalloproteinases (21.5\%) [57]. In order to perform rapid detection and quantification of venom antigens, commonly found in higher concentrations from wound swabs or wound aspirates (within 15-30 min after bite) compared to body fluids, commercial highly sensitive venom detection kits are available [58] but they cannot distinguish between venoms of closely related species. Moreover, the detection of venom in a wound swab is not enough evidence that the patient has been envenomed, then also serum and urine samples should be stored for enzyme immunoassays [59-62], liquid chromatography-mass spectrometry with time-of-fight (LC-TOF/MS), DNA fingerprinting, and antibody microarrays [63]. Highly specific methods such as the detection of venom gland mRNA by reverse-transcription PCR $[64,65]$ or snake-derived DNA in bite-wound swabs [66] are being developed.

Based on their effects, snake venoms can be classified as hemotoxic, neurotoxic, or cytotoxic and they can act all together involving multiple tissues and organs [51]. Neurotoxicity is a well-known effect of SBE by snakes of the Elapidae family (e.g., cobras, kraits, mambas, Australasian species, and sea snakes) but also of the Viperidae family (e.g., rattlesnakes, lance-headed pit vipers, and true vipers) [67]. Neurotoxic envenomation can cause a broad spectrum of early clinical manifestations varying from ptosis and ophthalmoplegia to paralysis of respiratory muscles, which causes asphyxia. Elapid venom can interfere with transmission across the motor end-plate as it can bind to the postsynaptic nicotinic receptors that would normally bind acetylcholine [14]. Delayed neurotoxic manifestations are represented by peripheral neuropathies, optic neuritis, and cortical blindness [68]. 
Cerebellar involvement, ataxia, and "locked-in syndrome" are very uncommon but they have been also described [68]. Outcomes are pretty good when prompt treatment has been provided.

Usually, snakes from the Viperidae family are considered hemotoxic [51]. Viperidae snakes were involved in most of the fatal SBE reviewed (28 cases out of 56 in total) followed soon after by Elapidae snakes ( 23 cases out of 56 ). Therefore, the 56 victims reviewed in this retrospective study suffered mostly hemotoxic and neurotoxic effects. Hemotoxic effects act on the heart and cardiovascular system and they can promote or inhibit hemostatic mechanisms including coagulation in different ways [53]. Hemotoxins can be pro-coagulant proteases such as prothrombin activators, thrombin-like enzymes, factor $\mathrm{X}$, and factor $\mathrm{V}$ activators but also anticoagulant proteases like the factor IX and X inhibitors, protein $\mathrm{C}$ activator, anticoagulant PLA2s [69]. The venom components acting on fibrinolysis are fibrinolytic enzymes and plasminogen activators [69]. Bleeding disorders can occur everywhere in the body from the bite site to the internal organs. Major complications related to hemotoxic effects include life-threatening diseases like acute kidney injury (AKI), ARDS, or its less serious variant acute lung injury (ALI), capillary leak syndrome (CLS), and DIC [70]. Victims suffering from cytotoxic envenoming are characterized by painful and progressive swelling at the bite site, developing further into blistering and bruising, which is sometimes coupled with systemic effects including hypovolemic shock [51]. Tissue necrosis is one of the major problems in patients with SBE [71]. Anaphylactic shock secondary to SBE has been reported in very few cases [72-74]. In suspected anaphylactic or anaphylactoid episodes related to drugs or venoms, a careful and complete review of the clinical history and the results of allergy testing might be useful in making the diagnosis or distinguishing them from other causes [75].

In the cases included in our review, the gross findings were non-specific. Autopsy findings reported are mostly non-specific pathologic changes consistent with a rapid death such as multi-visceral congestion, edema, and petechial hemorrhages. Signs of ischemic and hemorrhagic encephalopathy can be found mostly represented by intracranial or subarachnoid hemorrhages as well as myocardial hemorrhages. Widespread microthrombosis has been also described as the result of the coagulation disorders caused by the hemotoxic effects of SBE [76]. A relationship between SBE and myocardial infarction (MI) can also occur [77]. In particular, after SBE different factors such as coronary thrombosis due to the procoagulant factors in venom, direct toxic effect on cardiomyocytes, decreased oxygen-carrying capacity of blood due to hemolysis, coronary vasoconstriction brought about by endothelins and safatoxins in venom, and myocardial hemorrhage and microvascular thrombin deposition can determine an acute myocardial injury due to an imbalance between myocardial oxygen supply and demand $[77,78]$.

Other than pulmonary edema and congestion related to acute cardiac failure, pulmonary injuries by SBE are mostly represented by DAD and widespread microthrombosis in the DIC scenario. In particular, the effects of Crotalus durissus terrificus venom $(\mathrm{CdtV})$ on the pulmonary mechanic events have been studied in male swiss mice $[78,79]$. The histological analysis has reported perivascular/interstitial pulmonary edema $6 \mathrm{~h}$ after venom injection and pulmonary hemorrhages $12 \mathrm{~h}$ after. The microscopic quantitative analysis has also reported a significant increase of pulmonary inflammation 3 and $6 \mathrm{~h}$ after venom injection, returning to control values $24 \mathrm{~h}$ after [79]. The systemic inflammatory response and the mechanisms of pulmonary injuries by Crotalus durissus cascavella venom have been also investigated in mice by Azavedo et al. (2020) [80]. They reported a significant increase of peribronchial inflammatory infiltrates, emphysema, and focal atelectasis indicative of severe pulmonary inflammation $3 \mathrm{~h}$ after venom injection followed by a decrease in the activity of inflammatory response, despite an increase in vascular congestion, alveolar septal thickening, and emphysematous areas at $6 \mathrm{~h}$. Between 12 and $48 \mathrm{~h}$, there were perceptible inflammatory infiltrates in the pulmonary parenchyma, hemorrhagic focuses, vascular congestion, and bronchial muscle distention [80].

Acute nephrotoxicity is a relevant complication of SBE mostly represented by AKI that is a major cause of morbidity and mortality. AKI following SBE is mostly related to ATN or ACN and has multifactorial pathogenesis including, hypotension, coagulopathy, intravascular hemolysis, thrombotic 
microangiopathy (TMA), and DIC, capillary leak syndrome, rhabdomyolysis, complement activation, and sepsis [81]. Once developed ATN, AKI, and death can follow quickly [81,82]. A total of 22 cases out of a series of 121 snake bite-induced AKI have been studied histologically [83], 91\% of the study sample mostly after SBE by Viperidae family showed signs of ATN classified as severe in 55\% of the cases. Tubulointerstitial lesions represented, in particular, by ATN, were observed in $70 \%$ to $80 \%$ of patients with AKI [83]. However, kidney injuries have been also found in three cases of SBE by Bungarus Fasciatus, Elapidae family [3]. Among the intestinal injuries, bowel ischemia due to thrombosis of the mesenteric vessels has been reported in very few cases. [84,85]. Pituitary hemorrhage/necrosis and adrenal gland hemorrhage are additional autopsy findings reported [55].

\section{Conclusions}

SBE is a public health issue in tropical countries. The identification of snake species can be crucial for appropriate clinical management and can improve the prognosis. Although snake venom can be easily detected by toxicological analysis, diagnosis of SBE related death is challenging for the forensic pathologist. The cause of death from SBE is primarily related to the neurotoxic, cytotoxic, and hemotoxic effects of the venom components including the paralysis of respiratory muscles, which causes asphyxia and hypoxic-ischemic encephalopathy, hemorrhagic shock, cardiomyopathy, ATN, and death from anaphylactic shock [15]. Key elements are still represented by investigation of the bite mark and circumstantial data. Although rare in the European context, [86-88] it is important for the forensic pathologist to be aware of the details of the bite experience, local and systemic symptoms, and to check the bite marks by taking samples from bitten tissue, mostly located in the lower limbs.

\section{Materials and Methods}

The review of the literature was conducted independently by two examiners and carried out according to the PRISMA statement [10].

\subsection{Literature Search}

This retrospective study was performed using the following electronic databases: PubMed, Scopus, and Web of Science. The following keywords were searched in all fields: "snake bite" AND "autopsy" OR "snakebite" AND "autopsy" to recognize relevant research available until 18 April 2020. No time limit was set. The systematic search was further extended by snowball search and hand searching by using Google Scholar.

\subsection{Inclusion and Exclusion Criteria}

The following inclusion criterion was adopted: (1) papers reporting autopsy cases of fatal SBE with a description of the autopsy and/or histological findings. Exclusion criteria comprised the following: (1) animal studies; (2) scientific article not published in English, and (3) full text not available. For duplicate studies, only the article with more detailed information was included.

\subsection{Data Extraction}

Two examiners independently provided the initial selection of the articles to determine whether they might potentially fit the inclusion criteria. The title, abstract, and full text of each potentially pertinent study were reviewed. Disagreements on the eligibility of the studies were solved between the two examiners; if no agreement could be reached, it was resolved using a third co-author as a reviewer. A data extraction sheet was developed and for each selected study the following items were recorded: snake species; details of the victims (sex; age); location (country, working place); clinical history (time interval between snakebite and death; admission to hospital; administration of antivenom); autopsy features (morphology of the bite; macroscopic and microscopic features of the bite site; macroscopic and microscopic findings at autopsy). 
Author Contributions: Conceptualization, A.F., G.L.M. and. A.C.; methodology, P.Z., B.D.P. and C.P.C.; writing-original draft preparation, A.F., C.P.C. and A.C.; writing-review and editing, P.Z. and G.L.M.; supervision, B.D.P. and C.P.C. All authors have read and agreed to the published version of the manuscript.

Funding: This research received no external funding.

Acknowledgments: The costs of the open access publication were supported by the "Programma Valere 2020" of the University of Campania “Luigi Vanvitelli" (Naples, Italy).

Conflicts of Interest: The authors declare no conflict of interest.

\section{References}

1. Williams, D.J.; Faiz, M.A.; Abela-Ridder, B.; Ainsworth, S.; Bulfone, T.C.; Nickerson, A.D.; Habib, A.G.; Junghanss, T.; Fan, H.W.; Turner, M.; et al. Strategy for a globally coordinated response to a priority neglected tropical disease: Snakebite envenoming. PLoS Negl. Trop. Dis. 2019, 13, e0007059. [CrossRef] [PubMed]

2. WHO. Snakebite Envenoming. 2019. Available online: https://www.who.int/news-room/fact-sheets/detail/ snakebite-envenoming (accessed on 30 September 2020).

3. Sarkar, N.; Basu, S.; Chandra, P.; Chowdhuri, S.; Mukhopadhyay, P. Nephrotoxicity in krait bite: A rare case series of three fatalities in consecutive bites by a single snake. Egypt. J. Forensic Sci. 2018, 8, 12. [CrossRef]

4. Gutiérrez, J.M. Global Availability of Antivenoms: The Relevance of Public Manufacturing Laboratories. Toxins 2018, 11, 5. [CrossRef] [PubMed]

5. Laraba-Jebari, F.; Cherifi, F. Pathophysiological and Pharmacological Effects of Snake Venom Components: Molecular Targets. J. Clin. Toxicol. 2014, 4, 2. [CrossRef]

6. Liu, C.-C.; Yu, J.-S.; Wang, P.-J.; Hsiao, Y.-C.; Chen, Y.-C.; Lai, P.-F.; Hsu, C.-P.; Fann, W.-C.; Lin, C.-C. Development of sandwich ELISA and lateral flow strip assays for diagnosing clinically significant snakebite in Taiwan. PLoS Negl. Trop. Dis. 2018, 12, e0007014. [CrossRef]

7. WHO. Venomous Snakes Distribution and Species Risk Categories. 2010. Available online: https://apps.who. int/bloodproducts/snakeantivenoms/database/ (accessed on 30 September 2020).

8. WHO. Guidelines for the Production, Control and Regulation of Snake Antivenom Immunoglobulins. 2018. Available online: https://www.who.int/bloodproducts/snake_antivenoms/snakeantivenomguide/en/ (accessed on 28 September 2020).

9. Warrel, D.A. Guidelines for the Management of Snake Bite; World Health Organization: New Delhi, India, 2010.

10. Moher, D.; Liberati, A.; Tetzla, J.; Altman, D.G.; PRISMA Group. Preferred reporting items for systematicreviews and meta-analyses: The PRISMA statement. PLoS Med. 2009, 6, 264-269. [CrossRef]

11. Barraviera, B.; Júnior, J.C.B.; Arakaki, D.; Domingues, M.A.C.; Pereira, P.C.M.; Mendes, R.P.; Machado, J.M.; Meira, D.A.; Arkaki, D. A retrospective study of 40 victims of Crotalus snake bites: Analysis of the hepatic necrosis observed in one patient. Rev. Soc. Bras. Med. Trop. 1989, 22, 5-12. [CrossRef]

12. Benvenuti, L.A.; França, F.O.S.; Barbaro, K.C.; Nunes, J.R.; Cardoso, J.L.C. Pulmonary haemorrhage causing rapid death after Bothrops jararacussu snakebite: A case report. Toxicon 2003, 42, 331-334. [CrossRef]

13. Blaylock, R.S.M.; Tilbury, C.R.; Branch, W.R. Anaphylaxis following exposure to snake venoms in South Africa. Curr. Allergy Clin. Immunol. 2002, 15, 65-68.

14. Blumenthal, R.; Scholtz, P.E.P.; Shuttleworth, J.-L. Black Mamba Death: Venom versus Antivenom? Am. J. Forensic Med. Pathol. 2019, 40, 356-360. [CrossRef]

15. Chen, L.; Huang, G.-Z. Poisoning by toxic animals in China-18 autopsy case studies and a comprehensive literature review. Forensic Sci. Int. 2013, 232, e12-e23. [CrossRef]

16. Curry, S.C.; Kunkel, D.B. Toxicology rounds. Death from a rattlesnake bite. Am. J. Emerg. Med. 1985, 3, 227-235. [CrossRef]

17. Karlawad, M.; Nadaf, A.A.; Nayak, G.H. Snake bite at an unusual site-A case report. JKAMLS 2017, 26, 28-31.

18. Keith, J.; White, J.; Pascu, O.; Fabian, V. Fatal Cerebellar Hemorrhage Following Australian Brown Snake Envenomation. Can. J. Neurol. Sci. J. Can. des Sci. Neurol. 2012, 39, 120-121. [CrossRef] [PubMed]

19. Kitchens, C.S.; Hunter, S.; Van Mierop, L.H. Severe myonecrosis in a fatal case of envenomation by the canebrake rattlesnake (Crotalus horridus atricaudatus). Toxicon 1987, 25, 455-458. [CrossRef] 
20. Malbranque, S.; Barbey, C.; Smadja, D.; Courcier, D.; Warrell, D.A.; Piercecchi-Marti, M.D.; Thomas, L.; Bucher, B.; Ridarch, A. Fatal Diffuse Thrombotic Microangiopathy after a Bite by the "Fer-de-Lance" Pit Viper (Bothrops lanceolatus) of Martinique. Am. J. Trop. Med. Hyg. 2008, 78, 856-861. [CrossRef]

21. Malhotra, P.; Sharma, N.; Awasthi, A.; Vasishta, R.K. Fatal acute disseminated encephalomyelitis following treated snake bite in India. Emerg. Med. J. 2005, 22, 308-309. [CrossRef]

22. Marsh, N.; DeRoos, F.; Touger, M. Gaboon viper (Bitis gabonica) envenomation resulting from captive specimens-a review of five cases. Clin. Toxicol. 2007, 45, 60-64. [CrossRef] [PubMed]

23. McGarity, B.H.; Marshall, G.P.; A Loadsman, J.; Carr, S.J.; Harper, C.G. Fatal cerebral haemorrhage after tiger snake bite. Med. J. Aust. 1991, 155, 61-62. [CrossRef]

24. Junior, R.M. Snake bites by the jararacucu (Bothrops jararacussu): Clinicopathological studies of 29 proven cases in Sao Paulo State, Brazil. QJM Int. J. Med. 1997, 90, 323-334. [CrossRef]

25. Moar, J.J.; Hill, L.; Stewart, M. Histopatholological Findings in a Fatal Case of Rinkhals Envenomation: A New Forensic Pathology Finding? Am. J. Forensic Med. Pathol. 2016, 37, 236-240. [CrossRef] [PubMed]

26. Rathnayaka, R.N.; Ranathunga, P.N.; Ranaweera, J.; Jayasekara, K.; Kularatne, S. Cardiac arrest and atrial fibrillation in a patient after hump-nosed pit viper (Hypnale hypnale) bite. Toxicon 2018, 148, 33-39. [CrossRef]

27. Rathnayaka, R.N.; Ranathunga, P.; Kularatne, S. Systemic bleeding including pulmonary haemorrhage following hump-nosed pit viper (Hypnale hypnale) envenoming: A case report from Sri Lanka. Toxicon 2019, 170, 21-28. [CrossRef] [PubMed]

28. Rathnayaka, R.N.; Kularatne, S.; Kumarasinghe, K.; Ranaweera, J.; Ranathunga, P.N. Ischemic brain infarcts and intracranial haemorrhages following Russell's viper (Daboia russelii) bite in Sri Lanka. Toxicon 2017, 125, 70-73. [CrossRef]

29. Norris, R.L.; Pfalzgraf, R.R.; Laing, G. Death following coral snake bite in the United States-first documented case (with ELISA confirmation of envenomation) in over 40 years. Toxicon 2009, 53, 693-697. [CrossRef] [PubMed]

30. Okamoto, O.; Nakashima, R.; Yamamoto, S.; Hashimoto, T.; Takasaki, T.; Tokuda, H.; Sato, S.; Gamachi, A.; Hashimoto, H.; Inagaki, N. A lethal case of mamushi (Gloydius blomhoffii) bite: Severe bowel symptoms as a lethal sign. Acute Med. Surgery 2016, 4, 135-139. [CrossRef] [PubMed]

31. Paulis, M.G.; Faheem, A.L. Homicidal Snake Bite in Children. J. Forensic Sci. 2015, 61, 559-561. [CrossRef]

32. Kumar, G.P.; Arun, M.; Chandrakanth, H.; Balaraj, B. Uncommon combination of malignancy: Incidental diagnosis at forensic autopsy. Aust. J. Forensic Sci. 2013, 45, 49-53. [CrossRef]

33. Quain, R. Bite from the Hooded Snake, (Cobra de Capello) Rapid Death; Autopsy. Lancet 1852, 60, 397-400. [CrossRef]

34. Rathnayaka, R.N.; Ranathunga, P. Late onset of bilateral cerebral infarction following Russell's viper (Daboia russelii) bite. Sri Lankan J. Anaesthesiol. 2017, 25, 35. [CrossRef]

35. Silva, A.; Gamlaksha, D.; Waidyaratne, D. Medico-legal significance of the identification of offending snake in a fatal snake bite: A case report. J. Forensic Leg. Med. 2013, 20, 965-967. [CrossRef] [PubMed]

36. De Oliveira, S.S.; Freitas-De-Sousa, L.A.; Alves, E.C.; Ferreira, L.C.D.L.; Da Silva, I.M.; De Lacerda, M.V.G.; Fan, H.W.; Moura-Da-Silva, A.M.; Monteiro, W.M. Fatal stroke after Bothrops snakebite in the Amazonas state, Brazil: A case report. Toxicon 2017, 138, 102-106. [CrossRef]

37. Soe, S.; Win, M.M.; Htwe, T.T.; Lwin, M.; Thet, S.S.; Kyaw, W.W. Renal histopathology following Russell's viper (Vipera russelli) bite. Southeast Asian J. Trop. Med. Public Health 1993, 24, 193-197.

38. Sutherland, S.K. Deaths from snake bite in Australia, 1981-1991. Med. J. Aust. 1992, 157, 740-746. [CrossRef] [PubMed]

39. Than, T.; Francis, N.; Swe, T.-N.; Lwin, M.; Pe, T.; Soe, S.; Oo, M.-M.; Phillips, R.E.; Warrell, D.A. Contribution of focal haemorrhage and microvascular fibrin deposition to fatal envenoming by Russell's viper (Vipera russelli siamensis) in Burma. Acta Trop. 1989, 46, 23-38. [CrossRef]

40. Tibballs, J.; Henning, R.D.; Sutherland, S.K.; Kerr, A.R. Fatal cerebral haemorrhage after tiger snake (Notechis scutatus) envenomation. Med. J. Aust. 1991, 154, 275-276. [CrossRef]

41. Tilbury, C.R.; Verster, J. A fatal bite from the burrowing asp Atractaspis corpulenta (Hallowell 1854). Toxicon 2016, 118, 21-26. [CrossRef] 
42. Trancă, S.; Cocis, M.; Antal, O. Lethal case of Vipera bersus bite. Clujul Med. 2016, 89, 435-437. [CrossRef]

43. Varagunam, T.; Panabokke, R.G. Bilateral cortical necrosis of the kidneys following snakebite. Postgrad. Med. J. 1970, 46, 449-451. [CrossRef]

44. Wankhede, A.G. An unusual Russell's viper bite mark: A case report. Med. Sci. Law 2004, 44, 87-89. [CrossRef]

45. Warrell, D.; Barnes, H.; Piburn, M. Neurotoxic effects of bites by the Egyptian cobra (Naja haje) in Nigeria. Trans. R. Soc. Trop. Med. Hyg. 1976, 70, 78-79. [CrossRef]

46. O'Malley, B. Clinical Anatomy and Physiology of Exotic Species; Elsevier Saunders: Philadelphia, PA, USA, 2005.

47. Giugliano, P.; Feola, A.; La Sala, F.; Buonomo, C.; Della Pietra, B. A Case of suicide by a Large Number of sharp Force Injuries. Medico-Legal Updat. 2017, 18, 10. [CrossRef]

48. Gold, B.S.; Dart, R.C.; Barish, R.A. Bites of Venomous Snakes. N. Engl. J. Med. 2002, 347, 347-356. [CrossRef]

49. Luiselli, L.; Sale, L.; Akani, G.C.; Amori, G. Venomous Snake Abundance Within Snake Species' Assemblages Worldwide. Diversity 2020, 12, 69. [CrossRef]

50. Gutiérrez, J.M.; Theakston, R.D.G.; Warrell, D.A. Confronting the Neglected Problem of Snake Bite Envenoming: The Need for a Global Partnership. PLoS Med. 2006, 3, e150. [CrossRef]

51. Slagboom, J.; Kool, J.; Harrison, R.A.; Casewell, N.R. Haemotoxic snake venoms: Their functional activity, impact on snakebite victims and pharmaceutical promise. Br. J. Haematol. 2017, 177, 947-959. [CrossRef]

52. Zahradnicek, O.; Horáček, I.; Tucker, A.S. Viperous fangs: Development and evolution of the venom canal. Mech. Dev. 2008, 125, 786-796. [CrossRef]

53. Adukauskienè, D.; Varanauskienè, E.; Adukauskaitè, A. Venomous Snakebites. Medicina 2011, $47,461$. [CrossRef]

54. Hayes, W.K.; Corbit, A.G.; Cardwell, M.D.; Herbert, S.S. Interfang Distances of Rattlesnakes: Sexual, Interspecific, and Body Size-related Variation, and Implications for Snakebite Research and Management. Wilderness Environ. Med. 2017, 28, 101-107. [CrossRef]

55. Thein, C.M.; Byard, R.W. Characteristics and relative numbers of lethal snake bite cases in medicolegal practice in central Myanmar-A five year study. J. Forensic Leg. Med. 2019, 63, 52-55. [CrossRef]

56. Tasoulis, T.; Isbister, G.K. A Review and Database of Snake Venom Proteomes. Toxins 2017, 9, 290. [CrossRef]

57. Pereañez, J.A.; Preciado, L.M.; Fernández, J.; Camacho, E.; Lomonte, B.; Castro, F.; Cañas, C.A.; Galvis, C.; Castaño, S. Snake venomics, experimental toxic activities and clinical characteristics of human envenomation by Bothrocophias myersi (Serpentes: Viperidae) from Colombia. J. Proteom. 2020, 220, 103758. [CrossRef]

58. Sutherland, S.K. Rapid venom identification: Availability of kits. Med. J. Aust. 1979, 2, 602-603. [CrossRef] [PubMed]

59. Theakston, R.; Laing, G.D. Diagnosis of Snakebite and the Importance of Immunological Tests in Venom Research. Toxins 2014, 6, 1667-1695. [CrossRef]

60. Ho, M.; Warrell, M.; Warrell, D.; Bidwell, D.; Voller, A. A critical reappraisal of the use of enzyme-linked immunosorbent assays in the study of snake bite. Toxicon 1986, 24, 211-221. [CrossRef]

61. Van Dong, L. Immunogenicity of venoms from four common snakes in the South of Vietnam and development of ELISA kit for venom detection. J. Immunol. Methods 2003, 282, 13-31. [CrossRef]

62. Kulawickrama, S.; O’Leary, M.A.; Hodgson, W.C.; Brown, S.G.A.; Jacoby, T.; Davern, K.; Isbister, G.K. Development of a sensitive enzyme immunoassay for measuring taipan venom in serum. Toxicon 2010, 55, 1510-1518. [CrossRef]

63. Brunda, G.; Sashidhar, R.; Sarin, R. Use of egg yolk antibody (IgY) as an immunoanalytical tool in the detection of Indian cobra (Naja naja naja) venom in biological samples of forensic origin. Toxicon 2006, 48, 183-194. [CrossRef]

64. Chen, T.; Bjourson, A.J.; Orr, D.F.; Kwok, H.; Rao, P.; Ivanyi, C.; Shaw, C. Unmasking venom gland transcriptomes in reptile venoms. Anal. Biochem. 2002, 311, 152-156. [CrossRef]

65. Jia, Y.; Olvera, P.; Rangel, F.; Mendez, B.; Reddy, S. Rapid Identification of Phospholipase A2 Transcripts from Snake Venoms. Toxins 2019, 11, 69. [CrossRef]

66. Sharma, S.; Kuch, U.; Höde, P.; Bruhse, L.; Pandey, D.P.; Ghimire, A.; Chappuis, F.; Alirol, E. Use of Molecular Diagnostic Tools for the Identification of Species Responsible for Snakebite in Nepal: A Pilot Study. PLoS Negl. Trop. Dis. 2016, 10, e0004620. [CrossRef] 
67. Ranawaka, U.K.; Lalloo, D.G.; De Silva, H.J. Neurotoxicity in Snakebite-The Limits of Our Knowledge. PLoS Negl. Trop. Dis. 2013, 7, e2302. [CrossRef]

68. Chacko, A.; Andronikou, S.; Ramanjam, V. Hypoxic brain injury and cortical blindness in a victim of a Mozambican spitting cobra bite. S. Afr. J. Surg. 2015, 53, 67. [CrossRef]

69. Teixeira, C.; Fernandes, C.M.; Leiguez, E.; Chudzinski-Tavassi, A.M. Inflammation Induced by Platelet-Activating Viperid Snake Venoms: Perspectives on Thromboinflammation. Front. Immunol. 2019, 10, 2082. [CrossRef]

70. Gopalakrishnakone, P. Clinical Toxinology in Asia Pacific and Africa; Springer: Dordrecht, The Netherlands, 2015.

71. Liu, C.-C.; Chou, Y.-S.; Chen, C.-Y.; Liu, K.-L.; Huang, G.-J.; Yu, J.-S.; Wu, C.-J.; Liaw, G.-W.; Hsieh, C.-H.; Chen, C.-K. Pathogenesis of local necrosis induced by Naja atra venom: Assessment of the neutralization ability of Taiwanese freeze-dried neurotoxic antivenom in animal models. PLoS Negl. Trop. Dis. 2020, 14, e0008054. [CrossRef]

72. Hogan, D.E.; Dire, D.J. Anaphylactic shock secondary to rattlesnake bite. Ann. Emerg. Med. 1990, 19, 814-816. [CrossRef]

73. Marko, I. Çeko; Marko, S.; Alimehmeti, I.; Sulaj, Z. Anaphylactic Reactions due to Snakebite Vipera and Administration of Antivenom. Toxicol. Forensic Med. Open J. 2016, 1, 65-66. [CrossRef]

74. Rencher, L.; Schwartz, J.; Wilson, A. An Anaphylactoid Reaction to Rattlesnake Envenomation in a Pediatric Patient. Pediatr. Emerg. Care 2018, 34, e109-e110. [CrossRef] [PubMed]

75. Campobasso, C.P.; Procacci, R.; Caligara, M. Fatal Adverse Reaction to Ketorolac Tromethamine in Asthmatic Patient. Am. J. Forensic Med. Pathol. 2008, 29, 358-363. [CrossRef]

76. Chaudhary, S.C.; Sawlani, K.K.; Malhotra, H.S.; Singh, J. Snake bite-induced leucoencephalopathy. BMJ Case Rep. 2013, 2013, 2012007515. [CrossRef]

77. Kariyanna, P.T.; Jayarangaiah, A.; Kamran, H.; Schechter, J.; Soroka, S.; Amarnani, A.; Ray, J.; Yacoub, M.; Post, M.; Al-Bayati, S.; et al. Myocardial Infarction after Snakebite Envenomation: A Scoping Study. Scifed. J. Cardiol 2018, 2, 21.

78. Thygesen, K.; Alpert, J.S.; Jaffe, A.S.; Chaitman, B.R.; Bax, J.J.; Morrow, D.A.; White, H.D. The Executive Group on behalf of the Joint European Society of Cardiology (ESC)/American College of Cardiology (ACC)/American Heart Association (AHA)/World Heart Federation (WHF) Task Force for the Universal Definition of Myocardial Infarction Fourth Universal Definition of Myocardial Infarction (2018). Circulation 2018, 138, e618-e651. [CrossRef] [PubMed]

79. Nonaka, P.N.; Amorim, C.F.; Peres, A.C.P.; E Silva, C.A.M.; Zamuner, S.R.; Ribeiro, W.; Cogo, J.C.; Vieira, R.D.P.; Dolhnikoff, M.; De Oliveira, L.V.F. Pulmonary mechanic and lung histology injury induced by Crotalus durissus terrificus snake venom. Toxicon 2008, 51, 1158-1166. [CrossRef]

80. Azevedo, E.; Figueiredo, R.G.; Pinto, R.V.; Ramos, T.D.C.F.; Sampaio, G.P.; Santos, R.P.B.; Guerreiro, M.L.D.S.; Biondi, I.; Trindade, S.C. Evaluation of systemic inflammatory response and lung injury induced by Crotalus durissus cascavella venom. PLoS ONE 2020, 15, e0224584. [CrossRef]

81. Gopalakrishnan, N. Snake Envenoming-An Underreported Cause of Acute Kidney Injury. Kidney Int. Rep. 2019, 4, 643-646. [CrossRef]

82. Ramachandran, S.; Perera, M.V.F. Survival in renal cortical necrosis due to snake bite. Postgrad. Med. J. 1974, 50, 314-316. [CrossRef]

83. Vikrant, S.; Jaryal, A.; Parashar, A. Clinicopathological spectrum of snake bite-induced acute kidney injury from India. World J. Nephrol. 2017, 6, 150-161. [CrossRef]

84. Rosenthal, R.; Meier, J.; Koelz, A.; Muller, C.; Wegmann, W.; Vogelbach, P. Intestinal ischemia after bushmaster (Lachesis muta) snakebite-A case report. Toxicon 2002, 40, 217-220. [CrossRef]

85. Nault, J.-C.; Megarbane, B.; Theodore, J.; Deye, N.; Nemeth, J.; Valleur, P.; Baud, F.J. Poisoning-related bowel infarction: Characteristics and outcomes. Clin. Toxicol. 2009, 47, 412-418. [CrossRef]

86. Garkowski, A.; Czupryna, P.; Zajkowska, A.; Pancewicz, S.; Moniuszko, A.; Kondrusik, M.; Grygorczuk, S.; Gołębicki, P.; Letmanowski, M.; Zajkowska, J. Vipera berus bites in Eastern Poland-A retrospective analysis of 15 case studies. Ann. Agric. Environ. Med. 2012, 19, 793-797. 
87. Malina, T.; Krecsák, L.; Korsós, Z.; Takacs, Z. Snakebites in Hungary-Epidemiological and clinical aspects over the past 36 years. Toxicon 2008, 51, 943-951. [CrossRef]

88. Valenta, J.; Stach, Z.; Stritesky, M.; Michalek, P. Common Viper Bites in the Czech Republic-Epidemiological and Clinical Aspects during 15 Year Period (1999-2013). Prague Med. Rep. 2014, 115, 120-127. [CrossRef]

Publisher's Note: MDPI stays neutral with regard to jurisdictional claims in published maps and institutional affiliations.

(C) 2020 by the authors. Licensee MDPI, Basel, Switzerland. This article is an open access article distributed under the terms and conditions of the Creative Commons Attribution (CC BY) license (http://creativecommons.org/licenses/by/4.0/). 